\title{
EL PERSONAL DEL SISTEMA NACIONAL DE SALUD: ¿UNA REFUTACION DE LA JAULA DE HIERRO BUROCRATICA?
}

\section{Mauro F. Guillén}

\author{
Universidad de Yale
}

RESUMEN. El presente artículo analiza la distribución del personal del Instituto Nacional de la Salud. Se apuntan los factores que explican las disparidades provinciales y por Comunidades Autónomas. Se sugiere que, lejos de tener efectos homogeneizantes, la actuación del Estado y de las profesiones sanitarias ha rèsultado en una distribución de los recursos sanitarios sesgada hacia las instituciones, provincias y Comunidades Autónomas con mayor poder de influencia en las decisiones de asignación.

La reforma socialista diseña entre 1982 y 1986 un nuevo Sistema $\mathrm{Na}$ cional de Salud (SNS). En este SNS se integra el mayor organismo público de asistencia sanitaria, que es el Instituto Nacional de la Salud (INSALUD). El INSALUD posee la cuarta parte de las camas del país, y cuenta con más de 230.000 personas trabajando a su servicio. En las páginas que siguen se analiza la composición de ese personal según las distintas pirámides ocupativas, su distribución geográfica y los tipos de centros asistenciales donde trabajan. Los recursos de personal parecen concentrarse en las zonas más desarrolladas, y es allí donde el sector público sanitario es más importante. La distribución del personal parece ser el resultado de las acciones y presiones de las distintas burocracias públicas y de las profesiones que toman parte en el proceso de toma de decisiones sanitarias, sugiriendo un nuevo fenómeno de "Jaula de Hierro» burocrática de carácter no-isomórfico, pues ha provocado heterogeneidad entre las distintas Comunidades Autónomas 
(DiMaggio y Powell, 1983). En el análisis se utilizan los datos contenidos en las publicaciones anuales del INSALUD, tanto la Memoria como la Información económico-funcional de las instituciones sanitarias. Se concluye con el estudio de la distribución del personal de atención primaria de salud y del personal de consultas externas en los hospitales, mostrando las desigualdades y desequilibrios por Comunidades Autónomas. Dado el objetivo de SNS de cubrir a todos los habitantes, los cálculos se han realizado respecto de la población censal, y no de la asegurada.

\section{El personal del SNS}

El Instituto Nacional de la Salud (INSALUD), que había sido creado en 1978 a raíz de la reforma del Instituto Nacional de Previsión, iniciada dos años antes, concentra la mayor parte de los recursos públicos en asistencia sanitaria. La Ley General de Sanidad de 1986 crea un Sistema Nacional de Salud (SNS) que supone la unificación de todo el sector público sanitario.

EI INSALUD - que en 1984 cubre ya a un 91,6 por 100 de la población- posee la cuarta parte de las camas de hospital del país (51.928 en 1984), y atiende anualmente a 1.500 .000 personas, que suman más de 14.000.000 de estancias. En total, administra 130 centros hospitalarios propios y 13 hospitales ajenos (clínicos en su mayoría). Además, realiza atención primaria de salud (APS) en 1.000 centros (ambulatorios, consultorios, servicios de urgencia, centros de salud, etc.). El INSALUD es, en los años ochenta, una institución enorme, que cuenta con un presupuesto anual de más de un billón de pesetas (una tercera parte de los recursos de toda la Seguridad Social).

Los hospitales del INSALUD, junto con los dependientes del Ministerio de Educación y Ciencia (que a su vez se integran luego en el INSALUD), son los más dotados de personal del país. Dentro del SNS hay que distinguir entre instituciones cerradas (hospitales) e instituciones abiertas. En las primeras se presta asistencia sanitaria en régimen de internamiento, mientras que en las segundas la asistencia es ambulatoria. En los años ochenta existen cuatro tipos de instituciones cerradas: los centros especiales, las ciudades sanitarias, las residencias sanitarias, y las instituciones ajenas (administradas por el SNS, o concertadas).

Los centros especiales son -según el propio INSALUD - centros cuya función es la asistencia, investigación y perfeccionamiento de las técnicas sanitaria. Se trata de hospitales de gran tamaño, con mucho personal, y normalmente dotados de una tecnología compleja. En 1984 existen seis centros especiales: tres en Madrid y uno en Asturias, Cantabria y Toledo. Las ciudades sanitarias son complejos asistenciales compuestos por una residencia general y por otro hosptiales especiales que prestan una asistencia específica 
(Infantil, de Maternidad, etc.). Están situadas en zonas de alta densidad poblacional, y suelen concentrar los recursos públicos existentes para zonas geográficas amplias, causando a veces desplazamientos a los pacientes. En 1984 existen 12 ciudades sanitarias en España. Las residencias sanitarias (denominadas bospitales desde 1983) son hospitales generales de ámbito regional, provincial o comarcal. Son en total 112, distribuidas por toda la geografía del país. Las instituciones ajenas se dividen en instituciones ajenas administradas y financiadas por el INSALUD (IAAFI), y en instituciones concertadas, que en 1987 todavía no se habían integrado totalmente en el SNS. Las IAAFI son 13 en total, destacando los hospitales clínicos derivados de convenios con el Ministerio de Educación y Ciencia. Las instituciones concertadas son hospitales propiedad de otros sujetos públicos y privados en los que se presta asistencia sanitaria con cargo a los presupuestos del INSALUD, debido a la insuficiencia de los recursos de camas y personal del INSALUD para atender a toda la población protegida (especialmente en Cataluña y el País Vasco).

Las instituciones abiertas - 1.021 en 1984- aglutinan siete tipos de instituciones surgidas de las sucesivas iniciativas o reformas de la APS que se han producido en España: ambulatorios, consultorios, servicios de urgencia, servicios especiales de urgencia, centros de diagnóstico y tratamiento, dispensarios de accidentes de trabajo y enfermedades profesionales, así como los modernos centros de salud (que se crean para potenciar la atención primaria en las zonas de salud).

Los datos que a continuación se analizan proceden de las publicaciones anuales del INSALUD. En la Memoria anual (Instituto Nacional de la Salud, 1986) el personal del INSALUD aparece desglosado por tipos, Comunidades Autónomas y provincias. La distribución por tipo de institución y por tipo de personal se recoge anualmente en los tomos I y II de la Información económico-funcional de las instituciones sanitarias (Instituto Nacional de la Saiud, 1986). Desde 1981 no se recogen en ese informe los datos correspondientes a Cataluña.

En 1984 el Instituto Nacional de la Salud cuenta con unos recursos humanos de más de 230.000 personas, o un 64 por 100 del total de personas que trabajan en España en el sector sanitario. Esa cifra representa casi seis personas por cada 1.000 habitantes. El personal del INSALUD realiza en su mayoría funciones sanitarias ( 73 por 100), mientras que un 22 por 100 es personal no sanitario, y el 5 por 100 restante es personal de dirección y administración. Los datos exactos aparecen en la tabla 1.

La pirámide ocupativa del INSALUD se caracteriza porque un número reducido de personas es responsable de las tareas de planificación, dirección y control. La burocracia central del INSALUD ( 3.267 personas), ha realizado hasta ahora todas las funciones de planificación y de asignación de recursos. Unas 7.600 personas se reparten por las 50 Direcciones Provinciales del 


\section{TABLA 1}

Personal del INSALUD, en 1984

\begin{tabular}{|c|c|c|c|}
\hline Personal & Tolal ${ }^{1}$ & $\%$ & $\begin{array}{c}\text { Por } 100.000 \\
\text { babitantes: }\end{array}$ \\
\hline DIRECCION Y ADMINISTRACION ... & 10.903 & 4,8 & 28,4 \\
\hline $\begin{array}{lllllll}\text { Servicios Centrales } & \ldots & \ldots & \ldots & \ldots & \ldots & \ldots \\
\text { Direcciones Provinciales } & \ldots & \ldots & \ldots & \ldots\end{array}$ & $\begin{array}{l}3.267 \\
7.636\end{array}$ & $\begin{array}{l}1,1 \\
3,3\end{array}$ & $\begin{array}{r}8,5 \\
19,9\end{array}$ \\
\hline $\begin{array}{lllllllll}\text { SANITARIO } & \ldots & \ldots & \ldots & \ldots & \ldots & \ldots & \ldots & \ldots\end{array}$ & 166.075 & 72,7 & 432,6 \\
\hline $\begin{array}{llllllll}\text { Jerarquizado } & \ldots & \ldots & \ldots & \ldots & \ldots & \ldots & \ldots\end{array}$ & 22.780 & 10,0 & 59,3 \\
\hline 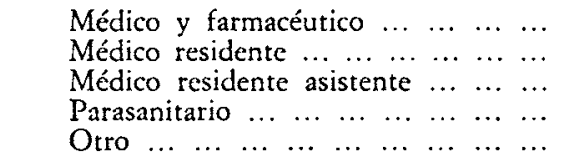 & $\begin{array}{r}16.411 \\
5.722 \\
337 \\
129 \\
181\end{array}$ & $\begin{array}{l}7,2 \\
2,5 \\
0,1 \\
0,1 \\
0,1\end{array}$ & $\begin{array}{r}42,8 \\
14,9 \\
0,9 \\
0,3 \\
0,5\end{array}$ \\
\hline Retribuido por bonorarios ... ... ... ... & 37.666 & 16,5 & 98,1 \\
\hline $\begin{array}{lllllll}\text { Medicina general } & \ldots & \ldots & \ldots & \ldots & \ldots & \ldots \\
\text { Médico especialista } & \ldots & \ldots & \ldots & \ldots & \ldots \\
\text { Médico ayudante especialista } & \ldots & \ldots \\
\text { Médico de urgencia } & \ldots & \ldots & \ldots & \ldots & \ldots\end{array}$ & $\begin{array}{r}15.571 \\
13.642 \\
4.285 \\
2.118\end{array}$ & $\begin{array}{l}7,7 \\
6,0 \\
1,9 \\
1,0\end{array}$ & $\begin{array}{r}40,6 \\
35,5 \\
11,2 \\
5,5\end{array}$ \\
\hline De enfermeria y auxiliar $\ldots \ldots \ldots$ & 91.940 & 40,2 & 239,5 \\
\hline 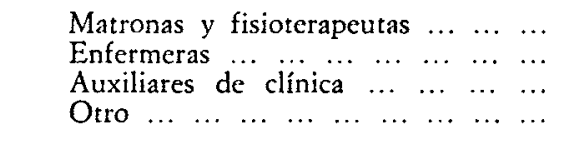 & $\begin{array}{r}3.349 \\
49.582 \\
38.387 \\
622\end{array}$ & $\begin{array}{r}1,5 \\
21,7 \\
16,8 \\
0,3\end{array}$ & $\begin{array}{r}8,7 \\
129,2 \\
100,0 \\
1,6\end{array}$ \\
\hline 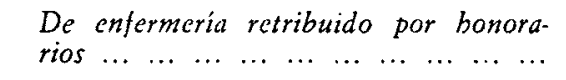 & 13.689 & 6,0 & 35,7 \\
\hline $\begin{array}{llllllll}\text { Practicantes } & \text { ATS } & \ldots & \ldots & \ldots & \ldots & \ldots & \ldots \\
\text { Practicantes } & \text { ATS } & \text { de } & \text { urgencia } & \ldots & \ldots \\
\text { Matronas } & \ldots & \ldots & \ldots & \ldots & \ldots & \ldots & \ldots \\
\end{array}$ & $\begin{array}{l}9.430 \\
1.732 \\
2.527\end{array}$ & $\begin{array}{l}4,1 \\
0,8 \\
1,1\end{array}$ & $\begin{array}{r}24,6 \\
4,5 \\
6,6\end{array}$ \\
\hline NO-SANITARIO & 52.522 & 22,5 & 136,8 \\
\hline 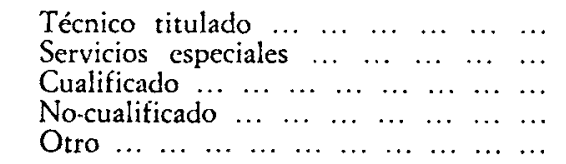 & $\begin{array}{r}901 \\
14.058 \\
12.776 \\
15.196 \\
8.591\end{array}$ & $\begin{array}{l}0,4 \\
6,2 \\
5,6 \\
6,7 \\
3,8\end{array}$ & $\begin{array}{r}2,3 \\
36,6 \\
33,3 \\
39,6 \\
22,4\end{array}$ \\
\hline $\begin{array}{llllllll}\text { TOTAL } & \ldots & \ldots & \ldots & \ldots & \ldots & \ldots & \ldots\end{array}$ & 228.500 & 100,0 & 595,3 \\
\hline
\end{tabular}

Notas: 'Incluye los ocupados en Ceuta (123) y Melilla (125) y, también, el personal sanitario ocupado en Servicios Centrales (63).

2 Según la población de derecho, calculada a 1 de julio de 1984 , de 38.386 .797 personas.

Fuente: Instituto Nacional de la Salud, Memoria 1984 (Madrid: INSALUD, 1984), pp. $49-80$. 
INSALUD. Esas 11.000 personas, que son funcionarios de la Seguridad Social, son quienes gestionan el funcionamiento de todo el sistema de asistencia sanitaria; y no sólo la asignación de recursos sanitarios, sino también la administración del sistema público de protección de la salud.

El personal sanitario - que reúne a un colectivo de 166.000 personasestá sometido a un régimen estatutario en el que caben principalmente dos modalidades de vinculación: jerarquizado y retribuido a través del servicio de honorarios. El personal jerarquizado está sometido a una dedicación total, mientras que el personal retribuido a través del servicio de honorarios se dedica de modo parcial. El personal médico tiene mayoritariamente una dedicación parcial, mientras que el de enfermería y auxiliar mantiene una dedicación total en casi nueve de cada diez casos. El personal de enfermería del INSALUD es - junto con el del Ministerio de Educación y Ciencia- el más especializado dentro de los hospitales españoles. El personal médico también ha alcanzado en el INSALUD un grado de especialización elevado (Nadal, 1984). Además, una característica importante es que hay más personal de enfermería titulado que auxiliar de enfermería. Ese fenómeno es consecuencia de dos factores: el sistema público no parece sustituir personal caro por personal más barato, como ocurre en el sector privado; y el sector público tiene tendencia a crear un grado de especialización más elevado que el realmente existente (las estadísticas reflejan no obstante esa especialización aparente).

El personal no sanitario representa una cuarta parte del total. En los últimos diez años ha aumentado considerablemente el peso relativo del personal técnico titulado y del personal de servicios especiales (unas 15.000 personas en 1984). Ambos tipos de personal realizan funciones de gestión y de administración, comprobándose así el proceso de especialización administrativa del personal no sanitario común a todo el sector hospitalario español.

La mayor parte del personal del INSALUD trabaja en hospitales. En 1984 el 86 por 100 del personal que presta sus servicios de asistencia sanitaria lo hace en instituciones cerradas, mientras que el 14 por 100 restante lo hace en centros de atención primaria de salud (APS). Además, un 7 por $100 \mathrm{del}$ total de personal está adscrito a los seis centros especiales en funcionamiento. Las 12 ciudades sanitarias cuentan con un 29 por 100 del personal total, mientras los hospitales absorben un 40 por 100 (tabla 2. Esas cifras sugieren que la distribución regional del personal del INSALUD es bastante desigual, pues más de una tercera parte del personal de asistencia sanitaria ( 36 por 100) trabaja en 16 complejos hospitalarios (los centros especiales y las ciudades sanitarias).

La mayor parte del personal de APS se concentra en los ambulatorios ( 38 por 100 del total de personal de asistencia primaria), y en los consultorios (10 por 100). El INSALUD dispone de 58 personas trabajando en APS 


\section{TABLA 2}

\section{Personal del INSALUD según el tipo de institución, en 1984 (Por cada 100.000 habitantes)}

\begin{tabular}{|c|c|c|}
\hline Tipo de institución & Tasa & $\%$ \\
\hline $\begin{array}{llllllllllllllll}\text { Hospitales } & \ldots & \ldots & \ldots & \ldots & \ldots & \ldots & \ldots & \ldots & \ldots & \ldots & \ldots & \ldots\end{array}$ & 419,7 & 86,0 \\
\hline 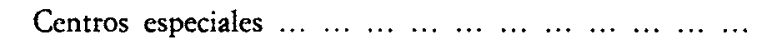 & 32,0 & 6,6 \\
\hline $\begin{array}{lllllllllll}\text { Ciudades sanitarias } & \ldots & \ldots & \ldots & \ldots & \ldots & \ldots & \ldots & \ldots & \ldots & \ldots\end{array}$ & 139,8 & 28,6 \\
\hline $\begin{array}{llllllllllllll}\text { Generales } & \ldots & \ldots & \ldots & \ldots & \ldots & \ldots & \ldots & \ldots & \ldots & \ldots & \ldots & \ldots & \ldots\end{array}$ & 186,9 & 38,3 \\
\hline $\begin{array}{lllllllllllllll}\operatorname{IAAFI}^{\prime} & \ldots & \ldots & \ldots & \ldots & \ldots & \ldots & \ldots & \ldots & \ldots & \ldots & \ldots & \ldots & \ldots & \ldots\end{array}$ & 61,1 & 12,5 \\
\hline $\begin{array}{llllllllllll}\text { Atención primaria } & \ldots & \ldots & \ldots & \ldots & \ldots & \ldots & \ldots & \ldots & \ldots & \ldots & \ldots\end{array}$ & 68,4 & 14,0 \\
\hline Centros de diagnóstico y tratamiento $\ldots \ldots \ldots \ldots$ & 1,9 & 0,4 \\
\hline 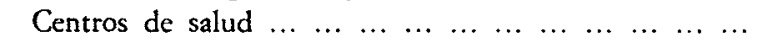 & 0,6 & 0,1 \\
\hline $\begin{array}{lllllllllllll}\text { Ambulatorios } & \ldots & \ldots & \ldots & \ldots & \ldots & \ldots & \ldots & \ldots & \ldots & \ldots & \ldots & \ldots\end{array}$ & 45,5 & 9,3 \\
\hline $\begin{array}{lllllllllllll}\text { Consultorios } & \ldots & \ldots & \ldots & \ldots & \ldots & \ldots & \ldots & \ldots & \ldots & \ldots & \ldots & \ldots\end{array}$ & 12,1 & 2,5 \\
\hline $\begin{array}{lllllllllll}\text { Servicios de } u r g e n c i a & \ldots & \ldots & \ldots & \ldots & \ldots & \ldots & \ldots & \ldots & \ldots & \ldots\end{array}$ & 7,9 & 1,6 \\
\hline $\begin{array}{lllllllllllll}\text { Dispensarios } & \ldots & \ldots & \ldots & \ldots & \ldots & \ldots & \ldots & \ldots & \ldots & \ldots & \ldots & \ldots\end{array}$ & 0,5 & 0,1 \\
\hline 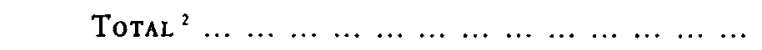 & 488,2 & 100,0 \\
\hline
\end{tabular}

Notas: Las tasas se han calculado a partir de la población de derecho, calculada a 1 de julio de 1984 , de 32.367 .636 personas (excluida Cataluña).

Instituciones ajenas administradas y financiadas por el INSALUD.

2 Incluye Ceuta y Melilla. No incluye Cataluña, para la que se carece de datos.

Fuente: Instituto Nacional de la Salud, Información económico-funcional de las instituciones sanitarias, 1984. Tomo I (Madrid: INSALUD, 1986), pp. 93, 101, 109, 117 y 125 .

por cada 100.000 habitantes. Esa tasa es reducida para los estándares europeos (Borgoño et al., 1985; De Miguel, 1985; OECD, 1985), aunque conviene tener en cuenta que el personal de hospitales también realiza tareas de atención primaria en las llamadas consultas externas. Una característica esencial es la heterogénea distribución espacial del personal del INSALUD.

\section{Distribución regional}

La distribución de recursos entre las distintas Comunidades Autónomas, y entre las distintas provincias, no es uniforme. A finales de los años ochenta, en un momento en que se están produciendo las transferencias en materia de sanidad (Andalucía, Cataluña, la Comunidad Valenciana y el País Vasco 
ya las poseen), la situación de partida de las Comunidades se convierte en una variable importante si se pretende reducir las tensiones territoriales españolas. En el mapa 1 se refleja la dotación de personal del INSALUD según la población de cada provincia. No parece existir una relación clara

\section{MAPA 1}

Personal del INSALUD, en 1984

(Tasas por 100.000 habitantes)

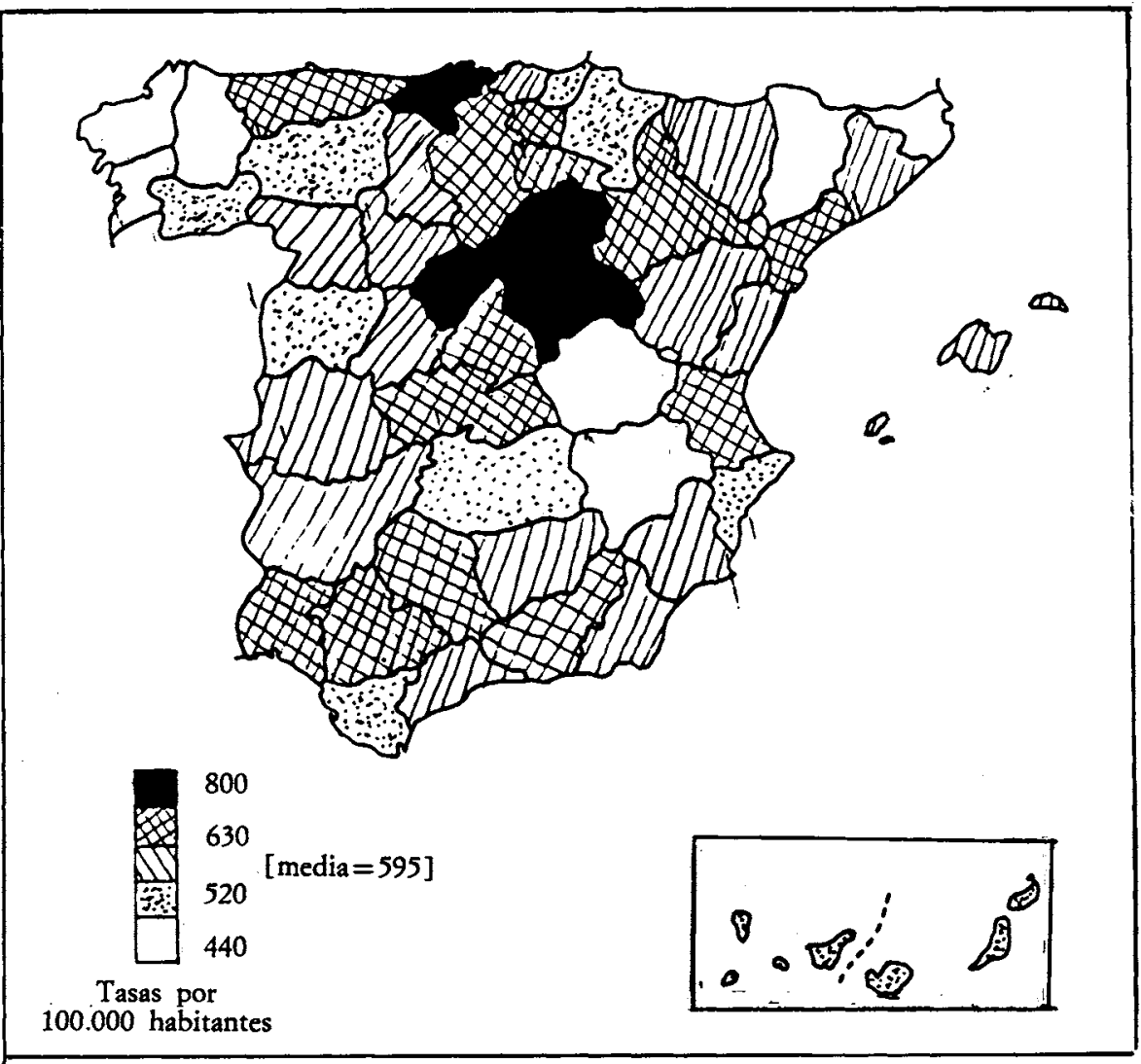

Nota: Las tasas se han calculado a partir de la población de derecho, calculada al 1 de julio de 1984 , que es de 38.386 .797 personas.

Fuente: Instituto Nacional de la Salud, Memoria 1984 (Madrid: INSALUD, 1984), pp. $53-80$. 
entre recursos públicos de personal y nivel de desarrollo de las provincias. Esa apreciación se ejemplifica con los casos de las tres provincias vascas, Navarra y, sobre todo, con Cataluña. Esas tres Comunidades Autónomas tienen relativamente poco personal sanitario del INSALUD, ya que tienen un alto grado de concertación de la asistencia sanitaria. Además de la importancia relativa del sector público sanitario conviene tener en cuenta que en la tasa de personal por habitante no sólo influyen los incrementos de personal, sino también la dinámica poblacional. Así, los casos de Avila, Soria y Guadalajara pueden explicarse más fácilmente. Las relaciones entre esas tres variables - nivel de desarrollo, importancia relativa del sector público sanitario, y movimientos poblacionales centro-periferia- explican la distribución que aparece en el mapa 1. Las regiones más desarrolladas son las que cuentan con más personal. La asociación entre subdesarrollo y escasa dotación de personal es más nítida si se observan las provincias más pobres: Ciudad Real, Cádız, Almería y, sobre todo, Pontevedra, La Coruña, Lugo, Albacete y Cuenca. La propia organización del sistema público hospitalario parece haber influido en esa distribución: la existencia de un centro especial o de una ciudad sanitaria hace que una determinada provincia alcance tasas más elevadas que las limítrofes: Santander, Asturias, Zaragoza, Valencia, Toledo, Sevilla.

Las diferencias de dotación se extienden a todos los tipos de personal (tabla 3). Las Comunidades Autónomas con mayor provisión de personal de dirección y administración son a su vez las que tienen más camas públicas: Cantabria, Aragón y Asturias. Es sorprendente el caso de Cataluña, que siendo una de las Comunidades con menor proporción de camas del INSALUD es, sin embargo, la que tiene más directivos y administradores (26 por cada 100.000 habitantes). Normalmente las Comunidades Autónomas con pocas camas del INSALUD son también las que tienen poco personal (INSALUD, 1985: 18).

El personal médico, y el de enfermería, siguen una distribución paralela por Comunidades Autónomas. Cuando existen muchos médicos también existen muchas enfermeras y auxiliares. La relación parece ser exponencial: las Comunidades con más personal total son, además, las que tienen más enfermeras en relación al número de médicos. Esto se puede observar en la primera columna de los datos siguientes: 


\begin{tabular}{|c|c|c|}
\hline Comunidad & $\begin{array}{l}\text { Personal } \\
\text { de enfermeria } \\
\text { por personal } \\
\text { médico }\end{array}$ & $\begin{array}{c}\% \text { del personal } \\
\text { no-sanitario que es } \\
\text { técnico titulado o de } \\
\text { servicios especiales }\end{array}$ \\
\hline $\begin{array}{llllllllllll}\text { Madrid } \ldots & \ldots & \ldots & \ldots & \ldots & \ldots & \ldots & \ldots & \ldots & \ldots & \ldots\end{array}$ & 1,6 & 32 \\
\hline $\begin{array}{lllllllllll}\text { Cataluña } & \ldots & \ldots & \ldots & \ldots & \ldots & \ldots & \ldots & \ldots & \ldots & \ldots\end{array}$ & 1,5 & 37 \\
\hline $\begin{array}{llllllllll}\text { Paîs } V a s c o & \ldots & \ldots & \ldots & \ldots & \ldots & \ldots & \ldots & \ldots & \ldots\end{array}$ & 1,6 & 28 \\
\hline $\begin{array}{llllllllllll}\text { Baleares } & \ldots & \ldots & \ldots & \ldots & \ldots & \ldots & \ldots & \ldots & \ldots & \ldots\end{array}$ & 2,0 & 28 \\
\hline $\begin{array}{lllllllllll}\text { Cantabria } & \ldots & \ldots & \ldots & \ldots & \ldots & \ldots & \ldots & \ldots & \ldots & \ldots\end{array}$ & 2,4 & 32 \\
\hline $\begin{array}{llllllllllll}\text { Navarra } & \ldots & \ldots & \ldots & \ldots & \ldots & \ldots & \ldots & \ldots & \ldots & \ldots\end{array}$ & 1,5 & 29 \\
\hline $\begin{array}{lllllllllll}\text { La Rioja } & \ldots & \ldots & \ldots & \ldots & \ldots & \ldots & \ldots & \ldots & \ldots & \ldots\end{array}$ & 1,7 & 28 \\
\hline $\begin{array}{llllllllllll}\text { Aragón } & \ldots & \ldots & \ldots & \ldots & \ldots & \ldots & \ldots & \ldots & \ldots & \ldots\end{array}$ & 2,0 & 29 \\
\hline $\begin{array}{llllllllll}\text { Valenciana } & \ldots & \ldots & \ldots & \ldots & \ldots & \ldots & \ldots & \ldots & \ldots\end{array}$ & 1,8 & 27 \\
\hline $\begin{array}{llllllllllll}\text { Asturias } & \ldots & \ldots & \ldots & \ldots & \ldots & \ldots & \ldots & \ldots & \ldots & \ldots\end{array}$ & 1,9 & 26 \\
\hline $\begin{array}{lllllllllll}\text { Canarias } & \ldots & \ldots & \ldots & \ldots & \ldots & \ldots & \ldots & \ldots & \ldots & \ldots\end{array}$ & 2,0 & 22 \\
\hline $\begin{array}{llllllllll}\text { Castilla y León } & \ldots & \ldots & \ldots & \ldots & \ldots & \ldots & \ldots & \ldots\end{array}$ & 1,5 & 20 \\
\hline $\begin{array}{lllllllllll}\text { Galicia } & \ldots & \ldots & \ldots & \ldots & \ldots & \ldots & \ldots & \ldots & \ldots & \ldots\end{array}$ & 1,6 & 23 \\
\hline $\begin{array}{lllllllllll}\text { Murcia } & \ldots & \ldots & \ldots & \ldots & \ldots & \ldots & \ldots & \ldots & \ldots & \ldots\end{array}$ & 2,0 & 26 \\
\hline $\begin{array}{llllllllllll}\text { Andalucía } & \ldots & \ldots & \ldots & \ldots & \ldots & \ldots & \ldots & \ldots & \ldots & \ldots\end{array}$ & 2,2 & 30 \\
\hline $\begin{array}{lllllllll}\text { Castilla-La Mancha } & \ldots & \ldots & \ldots & \ldots & \ldots & \ldots & \ldots\end{array}$ & 1,6 & 21 \\
\hline $\begin{array}{lllllllll}\text { Extremadura } & \ldots & \ldots & \ldots & \ldots & \ldots & \ldots & \ldots & \ldots\end{array}$ & 1,8 & 23 \\
\hline Total de España $\ldots \begin{array}{llllll} & \ldots & \ldots & \ldots & \ldots & \ldots\end{array}$ & 1,7 & 29 \\
\hline
\end{tabular}

Las Comunidades con una mayor dotación de personal total (por 100.000 habitantes) son también las que disponen de un mayor número de personal de enfermería por médico. Las Comunidades Autónomas con mayores tasas de camas de hospital del INSALUD tienen mayor dotación de personal de enfermería: Cantabria, Aragón y Austrias. Por el contrario, las regiones ricas con escasa presencia del sector público tienen tasas reducidas de ese personal (Navarra), así como las regiones pobres igualmente desprovistas de camas públicas (Galicia).

El personal no-sanitario es más abundante en aquellas Comunidades con centros o complejos hospitalarios mayores (Madrid, Cantabria, Aragón, Asturias, Murcia). Además, el proceso de especialización administrativa es más intenso en esas Comunidades: el porcentaje que representa el personal técnico titulado y el de servicios especiales es el más elevado (véase la segunda columna de los datos anteriores). Un caso peculiar lo constituye Cataluña, que dispone de la tasa más elevada de personal de dirección y administración en Direcciones Provinciales, y, además, un 37 por 100 de su personal no-sanitario se dedica también a tareas administrativas (frente al 29 por 100 en el total de España).

Estas relaciones entre los tres tipos de personal —médico, de enfermería 
TABLA 3

Personal del INSALUD por Comunidades Autónomas, en 1984

(Por cada 100.000 habitantes)

\begin{tabular}{|c|c|c|c|c|c|c|c|c|c|c|}
\hline Personal & Toral & $\begin{array}{l}\text { Coinunidad } \\
\text { de Madrid }\end{array}$ & Caraluña & $\begin{array}{l}\text { Pais } \\
\text { Vasco }\end{array}$ & Baleares & Cantabria & Navarra & La Rioja & Aragón & $\begin{array}{c}\text { Comunidad } \\
\text { Valenciana }\end{array}$ \\
\hline DIRECCION Y ADMINISTRACION. & 28,4 & 20,4 & 26,0 & 18.8 & 19,6 & 25,1 & 18,4 & 25,8 & 29,2 & 16,0 \\
\hline $\begin{array}{llllll}\text { Servicios Centrales } & \ldots & \ldots & \ldots & \ldots & \ldots \\
\text { Direcciones Provinciales } & \ldots & \ldots & \ldots & \ldots\end{array}$ & $\begin{array}{r}8.5 \\
19.9\end{array}$ & $\overline{20.4}$ & $\overline{26,0}$ & $\overline{18,8}$ & $\overline{19.6}$ & $\overline{25,1}$ & $\overline{18,4}$ & $\overline{25,8}$ & $\overline{29,2}$ & $\overline{16,0}$ \\
\hline $\begin{array}{llllllll}\text { SANITARIO } & \ldots & \ldots & \ldots & \ldots & \ldots & \ldots & \ldots\end{array}$ & 432,6 & 489,1 & 379,2 & 411,8 & 465,3 & 760,9 & 353,6 & 484,3 & 513,3 & 446,1 \\
\hline 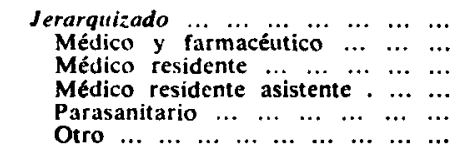 & $\begin{array}{r}59,3 \\
42,8 \\
14,9 \\
0,9 \\
0,3 \\
0,5\end{array}$ & $\begin{array}{r}77,3 \\
50,4 \\
25,1 \\
0,5 \\
0,3 \\
1,0\end{array}$ & $\begin{array}{r}46,5 \\
34,3 \\
10,7 \\
1,0 \\
0,1 \\
0,4\end{array}$ & $\begin{array}{l}61,2 \\
39,8 \\
21,3 \\
- \\
-., 1\end{array}$ & $\begin{array}{r}60,0 \\
45,4 \\
11,1 \\
2.1 \\
-1,5\end{array}$ & $\begin{array}{r}124,3 \\
69,3 \\
51.1 \\
3,6 \\
\frac{0,2}{}\end{array}$ & $\begin{array}{l}44,7 \\
27.6 \\
15.9 \\
- \\
\overline{1} .2\end{array}$ & $\begin{array}{l}59,3 \\
40,0 \\
\overline{19,2} \\
\overline{-}\end{array}$ & $\begin{array}{r}64,0 \\
49,6 \\
10,8 \\
1,8 \\
1,2 \\
0,6\end{array}$ & $\begin{array}{r}57,9 \\
44,1 \\
11,8 \\
1,0 \\
0,9 \\
0,2\end{array}$ \\
\hline 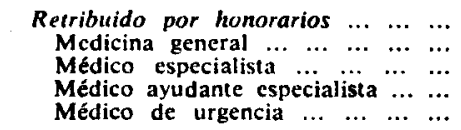 & $\begin{array}{r}98,1 \\
40,6 \\
35,5 \\
11,2 \\
5,5\end{array}$ & $\begin{array}{r}110,6 \\
43,8 \\
45,3 \\
16,7 \\
4,9\end{array}$ & $\begin{array}{r}105,3 \\
39,8 \\
47,4 \\
14,4 \\
3,8\end{array}$ & $\begin{array}{r}97,2 \\
44.3 \\
36,1 \\
8.7 \\
8,0\end{array}$ & $\begin{array}{r}96,7 \\
40,4 \\
38,3 \\
10,8 \\
7,2\end{array}$ & $\begin{array}{r}101,5 \\
46,0 \\
35,6 \\
12,8 \\
7,1\end{array}$ & $\begin{array}{r}96.5 \\
46,2 \\
34,6 \\
11.4 \\
4,3\end{array}$ & $\begin{array}{r}117,0 \\
88,2 \\
13,5 \\
10,8 \\
4,6\end{array}$ & $\begin{array}{r}109,5 \\
61,8 \\
32,1 \\
11,0 \\
4,6\end{array}$ & $\begin{array}{r}101,2 \\
43,5 \\
38,3 \\
10,7 \\
8,7\end{array}$ \\
\hline 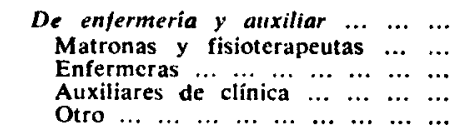 & $\begin{array}{r}239,5 \\
8.7 \\
129,2 \\
100,0 \\
1,6\end{array}$ & $\begin{array}{r}270,9 \\
7,6 \\
169,2 \\
92,6 \\
1,5\end{array}$ & $\begin{array}{r}193,3 \\
5,0 \\
104,4 \\
79,8 \\
4,1\end{array}$ & $\begin{array}{r}220,9 \\
15,5 \\
108,6 \\
96,5 \\
0,2\end{array}$ & $\begin{array}{r}270,1 \\
8.2 \\
127,5 \\
134,1 \\
0,1\end{array}$ & $\begin{array}{r}486,8 \\
12,3 \\
253,1 \\
221,5 \\
-\end{array}$ & $\begin{array}{r}175,9 \\
5,4 \\
98,8 \\
71,5 \\
0,2\end{array}$ & $\begin{array}{r}267,2 \\
9,2 \\
148,2 \\
109,7 \\
-\end{array}$ & $\begin{array}{r}302,8 \\
8,7 \\
161,0 \\
131,9 \\
1,2\end{array}$ & $\begin{array}{r}247,6 \\
8,2 \\
136,4 \\
102,7 \\
0,4\end{array}$ \\
\hline 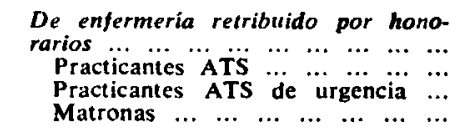 & $\begin{array}{r}35,7 \\
24,6 \\
4,5 \\
6,6\end{array}$ & $\begin{array}{r}10,3 \\
21,9 \\
2,4 \\
6,0\end{array}$ & $\begin{array}{r}34,1 \\
24,1 \\
3,4 \\
6,6\end{array}$ & $\begin{array}{r}32,5 \\
21,1 \\
5,8 \\
5,7\end{array}$ & $\begin{array}{r}38,5 \\
19,8 \\
8,7 \\
10,0\end{array}$ & $\begin{array}{r}48,3 \\
28,2 \\
7,1 \\
13,0\end{array}$ & $\begin{array}{r}36,5 \\
30,0 \\
4,3 \\
2,3\end{array}$ & $\begin{array}{r}40,8 \\
30,0 \\
4,6 \\
6,2\end{array}$ & $\begin{array}{r}37.1 \\
28.0 \\
3.0 \\
6.0\end{array}$ & $\begin{array}{r}39,3 \\
24,2 \\
5,9 \\
9,2\end{array}$ \\
\hline NO-SANITARIO & 136,8 & 148,4 & 115.7 & 113,7 & 152,8 & 245,6 & 78,5 & 151,7 & 152,3 & 139,1 \\
\hline 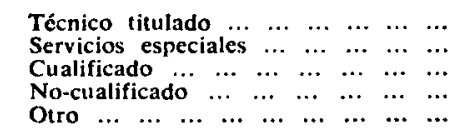 & $\begin{array}{r}2,3 \\
36,6 \\
33,3 \\
39.6 \\
22.4\end{array}$ & $\begin{array}{r}2,3 \\
44,8 \\
48,5 \\
38,4 \\
14,5\end{array}$ & $\begin{array}{r}1,6 \\
41,8 \\
27,3 \\
35,1 \\
9,9\end{array}$ & $\begin{array}{r}2,0 \\
30,1 \\
23,0 \\
39,2 \\
19,4\end{array}$ & $\begin{array}{r}1,9 \\
41,2 \\
64,1 \\
39,5 \\
6,1\end{array}$ & $\begin{array}{r}3,8 \\
73,6 \\
30,1 \\
70,1 \\
68,0\end{array}$ & $\begin{array}{r}\mathbf{0 , 8} \\
22,8 \\
33,4 \\
20.3 \\
1,2\end{array}$ & $\begin{array}{r}2,7 \\
40,0 \\
64,3 \\
43,5 \\
1,2\end{array}$ & $\begin{array}{r}2,2 \\
41,5 \\
29,0 \\
45,7 \\
34,0\end{array}$ & $\begin{array}{r}2.2 \\
36,0 \\
34,7 \\
39,2 \\
26,9\end{array}$ \\
\hline $\begin{array}{llllllll}\text { TOTAL } & \ldots & \ldots & \ldots & \ldots & \ldots & \ldots & \ldots\end{array}$ & 595,3 & 657,8 & 520,8 & 544.3 & 637,7 & 1.031 .6 & 450.4 & 661.7 & 694,9 & 601,2 \\
\hline
\end{tabular}


TABLA 3 (Continuación)

Personal del INSALUD por Comunidades Autónomas, en 1984 (Por cada 100.000 habitantes)

\begin{tabular}{|c|c|c|c|c|c|c|c|c|}
\hline Personal & Asturias & Canarias & $\begin{array}{l}\text { Castilla } \\
\text { y León }\end{array}$ & Galicia & Murcia & Andalueía & $\begin{array}{l}\text { Castilla- } \\
\text { La Mancha }\end{array}$ & Extremadura \\
\hline $\begin{array}{l}\text { DIRECCION Y ADMINISTRACION. } \\
\text { Servicios Centrales } \ldots \\
\text { Direcciones Provinciales }\end{array}$ & $\frac{26,1}{26,1}$ & $\frac{14,5}{14,5}$ & $\frac{23,4}{23,4}$ & $\frac{12,8}{12,8}$ & $\frac{15,7}{15,7}$ & $\frac{17,7}{17,7}$ & $\frac{17,4}{17,4}$ & $\frac{16,1}{16,1}$ \\
\hline 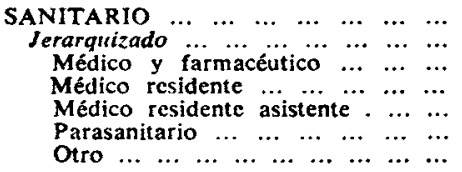 & $\begin{array}{r}522,0 \\
88,9 \\
63,2 \\
23,0 \\
0,9 \\
0,6 \\
1,2\end{array}$ & $\begin{array}{r}352,6 \\
44,2 \\
34,3 \\
9,3 \\
0,6 \\
0,1 \\
-\end{array}$ & $\begin{array}{r}451,0 \\
57,2 \\
41,9 \\
13,4 \\
1,2 \\
0,3 \\
0,3\end{array}$ & $\begin{array}{r}313,9 \\
42,2 \\
30,8 \\
10,6 \\
0,2 \\
0,2 \\
0,5\end{array}$ & $\begin{array}{r}479,7 \\
72,0 \\
47,3 \\
23,6 \\
0,4 \\
0,7\end{array}$ & $\begin{array}{r}449,4 \\
60,9 \\
46,5 \\
12,5 \\
0,8 \\
0,6 \\
0,5\end{array}$ & $\begin{array}{r}417,5 \\
51,2 \\
41,3 \\
8,1 \\
1,7 \\
0,1\end{array}$ & $\begin{array}{r}422,9 \\
58,1 \\
44,6 \\
11,7 \\
1,8 \\
0,1\end{array}$ \\
\hline 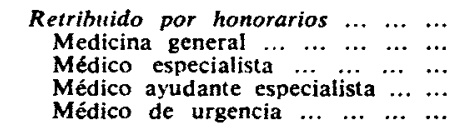 & $\begin{array}{r}93,4 \\
39,3 \\
36,5 \\
13,1 \\
4,5\end{array}$ & $\begin{array}{r}74,2 \\
31,3 \\
28,9 \\
8,8 \\
5,2\end{array}$ & $\begin{array}{r}126,8 \\
80,4 \\
31,9 \\
10,9 \\
3,6\end{array}$ & $\begin{array}{r}79,1 \\
42,7 \\
24,3 \\
7,4 \\
4,8\end{array}$ & $\begin{array}{l}88,0 \\
31,8 \\
33,7 \\
11,9 \\
10,5\end{array}$ & $\begin{array}{r}80,7 \\
37,4 \\
27,9 \\
8,9 \\
6,6\end{array}$ & $\begin{array}{r}112,3 \\
67,9 \\
34,4 \\
8,1 \\
1,9\end{array}$ & $\begin{array}{r}92,5 \\
57,6 \\
25,3 \\
5,6 \\
4,0\end{array}$ \\
\hline 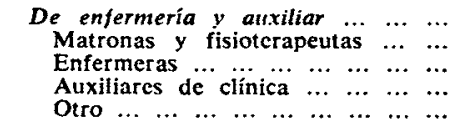 & $\begin{array}{r}306,4 \\
9,9 \\
164.8 \\
129,1 \\
2,6\end{array}$ & $\begin{array}{r}210,8 \\
9,3 \\
94,7 \\
106,2 \\
0,6\end{array}$ & $\begin{array}{r}224,6 \\
7,8 \\
123,5 \\
92,4 \\
0,9\end{array}$ & $\begin{array}{r}157,4 \\
12,4 \\
78,3 \\
66,1 \\
0,6\end{array}$ & $\begin{array}{r}276,1 \\
9,0 \\
124,7 \\
130,3 \\
12,0\end{array}$ & $\begin{array}{r}274,2 \\
9,5 \\
147,4 \\
116,7 \\
0,6\end{array}$ & $\begin{array}{r}207,1 \\
9,1 \\
104,3 \\
93,1 \\
0,7\end{array}$ & $\begin{array}{r}226,2 \\
8,5 \\
118,3 \\
98,9 \\
0,5\end{array}$ \\
\hline 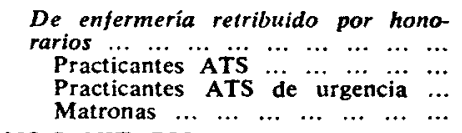 & $\begin{array}{r}30,9 \\
21,6 \\
4,5 \\
4,8\end{array}$ & $\begin{array}{r}23,5 \\
15,1 \\
5,9 \\
2,5\end{array}$ & $\begin{array}{r}42,5 \\
34,5 \\
3,2 \\
4,9\end{array}$ & $\begin{array}{r}35,2 \\
22,3 \\
4,7 \\
8,1\end{array}$ & $\begin{array}{r}4,3,6 \\
17,4 \\
21,2 \\
5,1\end{array}$ & $\begin{array}{r}33,6 \\
23,0 \\
3,9 \\
6,7\end{array}$ & $\begin{array}{r}46,9 \\
37,6 \\
1,8 \\
7,5\end{array}$ & $\begin{array}{r}46,1 \\
35,8 \\
3,9 \\
6,4\end{array}$ \\
\hline 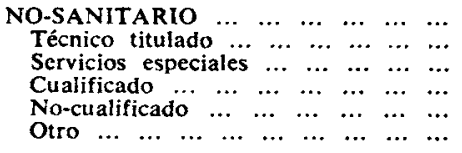 & $\begin{array}{r}178,1 \\
3,6 \\
42,0 \\
34,0 \\
54,3 \\
44,2\end{array}$ & $\begin{array}{r}109,9 \\
1,8 \\
22,3 \\
23,3 \\
37,4 \\
25,0\end{array}$ & $\begin{array}{r}124,6 \\
1,9 \\
25,5 \\
33,4 \\
35,9 \\
27,9\end{array}$ & $\begin{array}{r}100,9 \\
2,1 \\
20,7 \\
20,4 \\
30,8 \\
26,8\end{array}$ & $\begin{array}{r}132,1 \\
1,5 \\
33,0 \\
30,2 \\
45,0 \\
22,5\end{array}$ & $\begin{array}{r}153,1 \\
3,4 \\
43,4 \\
31,4 \\
44,6 \\
30,2\end{array}$ & $\begin{array}{r}122,2 \\
3,4 \\
23,1 \\
38,8 \\
39,4 \\
17,6\end{array}$ & $\begin{array}{r}144,1 \\
2,1 \\
30,7 \\
49,5 \\
44,5 \\
17,3\end{array}$ \\
\hline $\begin{array}{llllllll}\text { TOtAL } & \ldots & \ldots & \ldots & \ldots & \ldots & \ldots & \ldots\end{array}$ & 723,8 & 477,0 & 599,0 & 427,6 & 627,6 & 620,2 & 557,1 & 583,1 \\
\hline
\end{tabular}

Nota: Las Comunidades Autónomas aparecen ordenadas según la renta per capita en 1981. Banco de Bilbao, Renta nacional de España y su distribución provincial, 1981 (Bilbao: Banco de Bilbao, 1983), p. 76 . Baleares ocupa el segundo puesto en renta per capita, pero aparece cn cuarto lugar por
mayor afinidad en su grado de desarrollo sociosanitario. Las tasas se han calculado a partir de la población de derecho, calculada a 1 de julio de 1984, de 38.386 .797 personas.

Fuente: Instituto Nacional de la Salud. Memoria 1984 (Madrid: INSALUD, 1984), pp. 49-80. 
y no-sanitario- se observan mejor en la tabla 4. Las Comunidades Autónomas más desarrolladas, con un sector público sanitario mayor, y con centros hospitalarios de mayor tamaño y complejidad (centros especiales y ciudades sanitarias) tienen proporcionalmente más personal de enfermería y personal no-sanitario que personal médico (Cantabria, Aragón, Andalucía, Murcia, Baleares y Asturias). El caso contrario es el de Castilla y León, Cataluña, el País Vasco y Navarra, que cuentan con un mayor número de médicos en términos relativos. Cuanto más importante es el sistema público en una Comunidad, y cuanto mayor es el tamaño medio de los centros hospitalarios, mayor es también el número de personal de enfermería y no-sanitario respecto al de personal médico (véase el Gráfico 1).

\section{GRAFICO 1}

Personal del INSALUD por Comunidades Autónomas, en 1984 (En porcentajes del total)

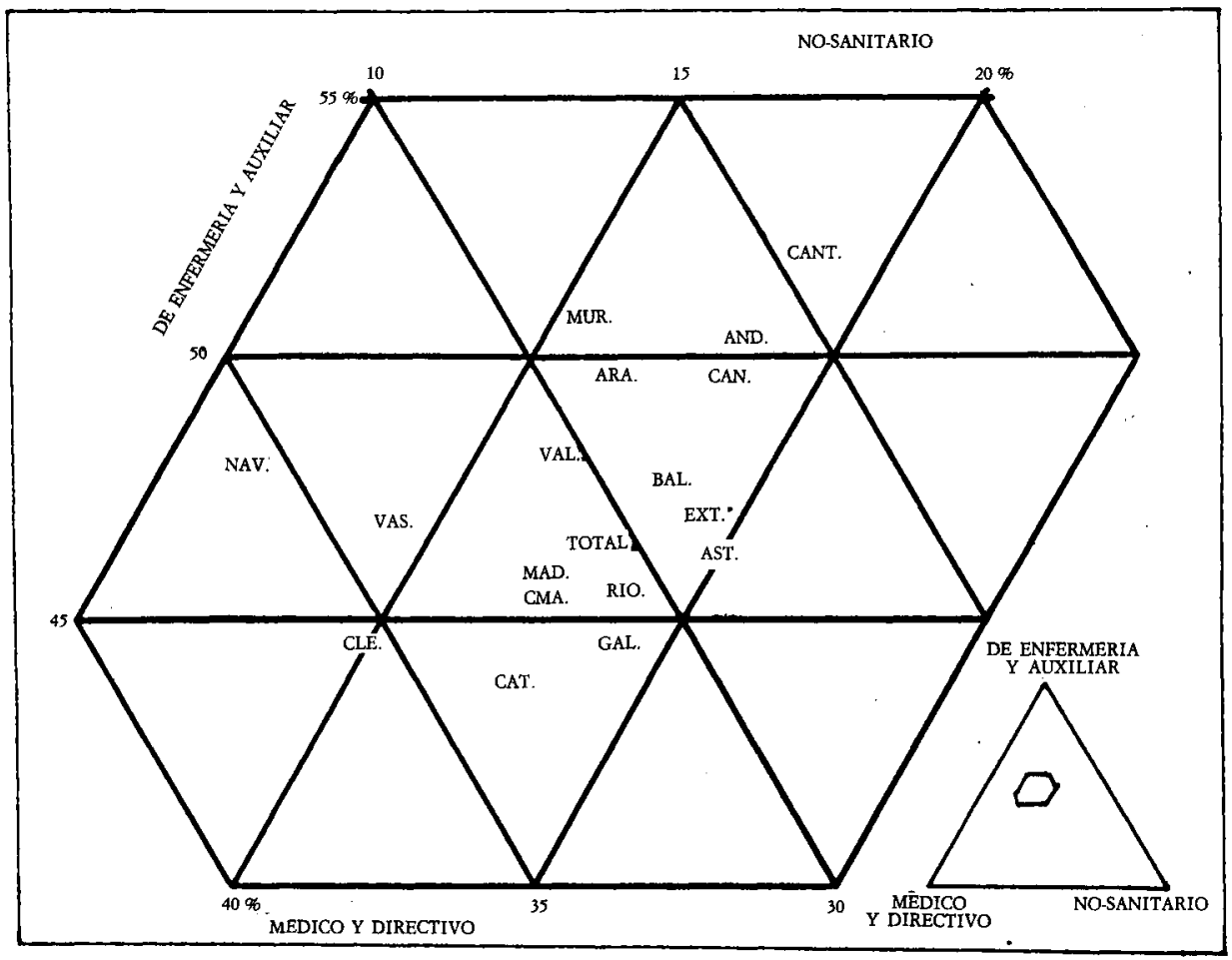

Fuente: Instituto Nacional de la Salud, Memoria 1984 (Madrid: INSALUD, 1984), pp. $49-80$. 
TABLA 4

Personal del INSALUD por Comunidades Autónomas, en 1984 (En porcentajes)

\begin{tabular}{|c|c|c|c|c|c|}
\hline \multirow[b]{2}{*}{ Comunidad Autónoma ${ }^{1}$} & \multirow[b]{2}{*}{ Total $(100 \%)$} & \multirow{2}{*}{$\begin{array}{l}\text { Personal de } \\
\text { dirección } y \\
\text { administracion }\end{array}$} & \multicolumn{2}{|c|}{ PERSONAL SANITARIO } & \multirow[b]{2}{*}{$\begin{array}{c}\text { Personal } \\
\text { no-sanitario }\end{array}$} \\
\hline & & & Médico & $\begin{array}{c}\text { De enfermeria } \\
\text { y auxiliar }\end{array}$ & \\
\hline 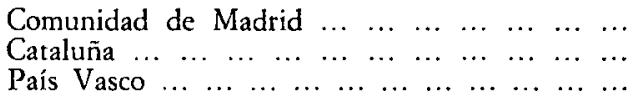 & $\begin{array}{l}31.730 \\
31.350 \\
11.775\end{array}$ & $\begin{array}{l}3,1 \\
5,0 \\
3,5\end{array}$ & $\begin{array}{l}28,6 \\
29,1 \\
29,0\end{array}$ & $\begin{array}{l}45,8 \\
43,7 \\
46,6\end{array}$ & $\begin{array}{l}22,6 \\
22,2 \\
20,9\end{array}$ \\
\hline 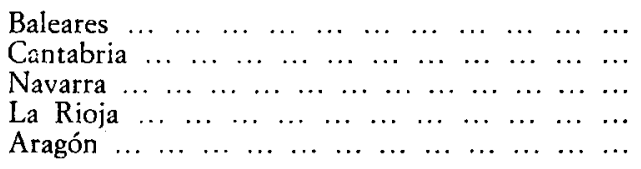 & $\begin{array}{l}4.260 \\
5.385 \\
2.330 \\
1.719 \\
8.398\end{array}$ & $\begin{array}{l}3,1 \\
2,4 \\
4,1 \\
3,9 \\
4,2\end{array}$ & $\begin{array}{l}24,5 \\
21,9 \\
31,3 \\
26,7 \\
25,0\end{array}$ & $\begin{array}{l}48,4 \\
51,9 \\
47,2 \\
46,5 \\
48,9\end{array}$ & $\begin{array}{l}24,0 \\
23,8 \\
17,4 \\
22,9 \\
21,9\end{array}$ \\
\hline 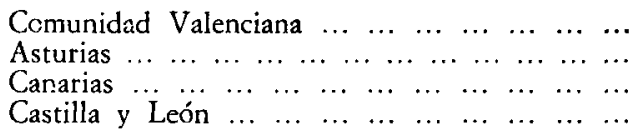 & $\begin{array}{r}22.460 \\
8.223 \\
6.745 \\
15.543\end{array}$ & $\begin{array}{l}2,7 \\
3,6 \\
3,0 \\
3,9\end{array}$ & $\begin{array}{l}26,5 \\
25,2 \\
24,9 \\
30,7\end{array}$ & $\begin{array}{l}47,7 \\
46,6 \\
49,1 \\
44,6\end{array}$ & $\begin{array}{l}23,1 \\
24,6 \\
23,0 \\
20,8\end{array}$ \\
\hline $\begin{array}{lllllllllllll}\text { Galicia } & \ldots & \ldots & \ldots & \ldots & \ldots & \ldots & \ldots & \ldots & \ldots & \ldots & \ldots & \ldots \\
\text { Murcia } & \ldots & \ldots & \ldots & \ldots & \ldots & \ldots & \ldots & \ldots & \ldots & \ldots & \ldots & \ldots \\
\text { Andalucía } & \ldots & \ldots & \ldots & \ldots & \ldots & \ldots & \ldots & \ldots & \ldots & \ldots & \ldots \\
\text { Castilla-La } & \text { Mancha } & \ldots & \ldots & \ldots & \ldots & \ldots & \ldots & \ldots & \ldots \\
\text { Extremadura } & \ldots & \ldots & \ldots & \ldots & \ldots & \ldots & \ldots & \ldots & \ldots & \ldots \\
\end{array}$ & $\begin{array}{r}12.181 \\
6.198 \\
41.080 \\
9.259 \\
6.280\end{array}$ & $\begin{array}{l}3,0 \\
2,5 \\
2,9 \\
3,1 \\
2,8\end{array}$ & $\begin{array}{l}28,4 \\
25,4 \\
22,8 \\
29,4 \\
25,8\end{array}$ & $\begin{array}{l}45,0 \\
51,0 \\
49,6 \\
45,6 \\
46,7\end{array}$ & $\begin{array}{l}23,6 \\
21,1 \\
24,7 \\
21,9 \\
24,7\end{array}$ \\
\hline $\begin{array}{llllllllllll}\text { TotaL }^{2} & \ldots & \ldots & \ldots & \ldots & \ldots & \ldots & \ldots & \ldots & \ldots & \ldots\end{array}$ & 228.500 & $4,8^{3}$ & 26,0 & 46,2 & 23,0 \\
\hline
\end{tabular}

Notas: ${ }^{1}$ Ordenadas según la renta per capila en 1981. Banco de Bilbao, Renta nacional de España y su distribución provincial, 1981 (Bilbao: Banco de Bilbao, 1983), p. 76. Baleares ocupa el segundo puesto en renta per capita, pero aparece dentro del segundo grupo de Comunidades Autónomas por mayor afinidad en su grado de desarrollo sociosanitario.

2 Incluye las personas ocupadas en Ceuta (123) y Melilla (125). En Servicios Centrales existen, además, 63 personas con función sanitaria.

3 Incluye, además, 3.267 personas en Servicios Centrales.

Fuente: Instituto Nacional de la Salud, Memoria 1984 (Madrid: INSALUD, 1984), pp. 49-80. 
Esa misma relación ocurre con la proporción de médicos en formación (MIR):

\begin{tabular}{|c|c|}
\hline Comunidad & $\begin{array}{l}\text { \% del total de } \\
\text { personal médico } \\
\text { que son MIR }\end{array}$ \\
\hline $\begin{array}{lllllllllll}\text { Madrid } & \ldots & \ldots & \ldots & \ldots & \ldots & \ldots & \ldots & \ldots & \ldots & \ldots \\
\text { Cataluña } & \ldots & \ldots & \ldots & \ldots & \ldots & \ldots & \ldots & \ldots & \ldots & \ldots \\
\text { País Vasco } & \ldots & \ldots & \ldots & \ldots & \ldots & \ldots & \ldots & \ldots & \ldots & \ldots\end{array}$ & $\begin{array}{r}13,6 \\
7,7 \\
13,4\end{array}$ \\
\hline 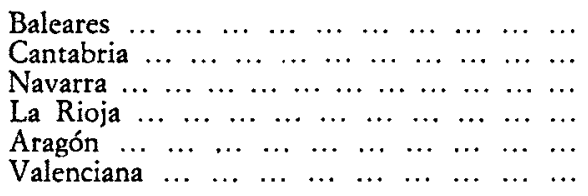 & $\begin{array}{r}8,4 \\
24,3 \\
11,2 \\
10,9 \\
7,3 \\
8,0\end{array}$ \\
\hline $\begin{array}{lllllllllll}\text { Asturias } & \ldots & \ldots & \ldots & \ldots & \ldots & \ldots & \ldots & \ldots & \ldots & \ldots \\
\text { Canarias } & \ldots & \ldots & \ldots & \ldots & \ldots & \ldots & \ldots & \ldots & \ldots & \ldots \\
\text { Castilla y } & \text { León } & \ldots & \ldots & \ldots & \ldots & \ldots & \ldots & \ldots & \ldots\end{array}$ & $\begin{array}{r}13,1 \\
8,3 \\
7,9\end{array}$ \\
\hline $\begin{array}{lllllllllll}\text { Galicia } & \ldots & \ldots & \ldots & \ldots & \ldots & \ldots & \ldots & \ldots & \ldots & \ldots \\
\text { Murcia } & \ldots & \ldots & \ldots & \ldots & \ldots & \ldots & \ldots & \ldots & \ldots & \ldots \\
\text { Andalucia } & \ldots & \ldots & \ldots & \ldots & \ldots & \ldots & \ldots & \ldots & \ldots & \ldots \\
\text { Castilla-La } & \text { Manch } & \ldots & \ldots & \ldots & \ldots & \ldots & \ldots & \ldots & \ldots \\
\text { Extremadura } & \ldots & \ldots & \ldots & \ldots & \ldots & \ldots & \ldots & \ldots & \ldots \\
\end{array}$ & $\begin{array}{r}8,9 \\
15,0 \\
9,4 \\
6,0 \\
8,9\end{array}$ \\
\hline Total de España & 10,0 \\
\hline
\end{tabular}

La distribución del personal del INSALUD por los distintos tipos de instituciones sigue dos pautas alternativas: 1) si una Comunidad posee abundancia de personal hospitalario suele disponer también de tasas elevadas de personal de APS; y 2) la distribución del personal entre los distintos tipos de instituciones abiertas y cerradas no sigue aparentemente un proceso estructural, sino que se amolda a la propia dinámica de creación de establecimientos (tabla 5). Las Comunidades Autónomas con abundantes recursos públicos (Madrid, Cantabria, Aragón, Asturias, Murcia y Andalucía) tienen tasas altas tanto de personal hospitalario como de APS. Es, además, en esas Comunidades donde hay más personal en instituciones ajenas.

Son varios los factores que explican la abundancia (o la escasez) relativa de personal del INSALUD en las Comunidades Autónomas: nivel de desarrollo, importancia relativa del sector público y tamaño y complejidad de los hospitales. Se pueden, pues, establecer cuatro grupos de Comunidades de parecido nivel de desarrollo socioeconómico en los que las disparidades de recursos públicos de personal son decrecientes conforme disminuye el nivel de desarrollo (mapa 2). El llamado triángulo industrial constituye el primer grupo, en el que se sitúan Madrid (con tasas elevadas de tipo tipo de per- 


\section{TABLA 5}

Personal del INSALUD según el tipo de institución, en 1984

(Por cada 100.000 habitantes)

\begin{tabular}{|c|c|c|c|c|c|c|c|c|c|c|c|c|c|}
\hline \multirow[b]{2}{*}{ Comunidad Autónoma ${ }^{1}$} & \multirow[b]{2}{*}{ Total } & \multicolumn{5}{|c|}{ HOSPITALES } & \multicolumn{7}{|c|}{ ATENCION PRIMARIA } \\
\hline & & Total & $\begin{array}{l}\text { Centros } \\
\text { espe- } \\
\text { ciales }\end{array}$ & $\begin{array}{l}\text { Ciuda- } \\
\text { des sani- } \\
\text { tarias }\end{array}$ & $\begin{array}{l}\text { Gene- } \\
\text { rales }\end{array}$ & $I A A F I^{2}$ & Total & $\begin{array}{l}\text { Centros } \\
\text { de diag- } \\
\text { nóstico } \\
\text { y trata- } \\
\text { miento }\end{array}$ & $\begin{array}{l}\text { Centros } \\
\text { de } \\
\text { salud }\end{array}$ & $\begin{array}{c}\text { Ambula- } \\
\text { torios }\end{array}$ & $\begin{array}{l}\text { Consul- } \\
\text { torios }\end{array}$ & $\begin{array}{l}\text { Servicios } \\
\text { de } \\
\text { urkencia }\end{array}$ & $\begin{array}{l}\text { Dispen- } \\
\text { sarios }\end{array}$ \\
\hline Comunidad de Madrid ... & 624,2 & 522,8 & 118,6 & 218,1 & 40,8 & 145,3 & 101,4 & 一 & 2,8 & 55,9 & 26,8 & 15,5 & 0,4 \\
\hline $\begin{array}{c}\text { Cataluña }{ }^{3} \\
\text { 'aís Vasco }\end{array}$ & 381,7 & 306,1 & $\stackrel{\cdots}{-}$ & 154,2 & 151,8 & $\underline{-}$ & 75,7 & 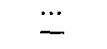 & - & 44,8 & 15,4 & 15,3 & 0,1 \\
\hline 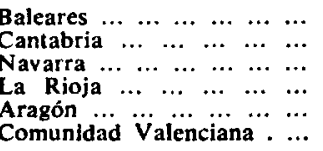 & $\begin{array}{l}466,6 \\
778,0 \\
278,2 \\
449,2 \\
685.7 \\
495,8\end{array}$ & $\begin{array}{l}405,2 \\
704,0 \\
223,5 \\
382,6 \\
606,3 \\
430,2\end{array}$ & $\begin{array}{l}\overline{704,0} \\
\overline{-} \\
-\end{array}$ & $\begin{array}{l}\bar{z} \\
\bar{z} \\
287,4 \\
159,5\end{array}$ & $\begin{array}{l}405,2 \\
\overline{223,5} \\
382,6 \\
145,4 \\
219,7\end{array}$ & $\begin{array}{l}\bar{Z} \\
\overline{173,5} \\
51,0\end{array}$ & $\begin{array}{l}61,4 \\
73,9 \\
54,7 \\
66,6 \\
79,4 \\
65,6\end{array}$ & $\begin{array}{l}E \\
E \\
-\end{array}$ & $\begin{array}{l}\overline{1,3} \\
\overline{-} \\
\overline{0,5}\end{array}$ & $\begin{array}{l}41,3 \\
52,1 \\
35,4 \\
53,5 \\
53,2 \\
41,2\end{array}$ & $\begin{array}{l}10,6 \\
14,6 \\
15,1 \\
10,4 \\
19,5 \\
14,9\end{array}$ & $\begin{array}{l}8,2 \\
5,7 \\
4,3 \\
2,7 \\
6,0 \\
8,8\end{array}$ & $\begin{array}{l}1,2 \\
0,2 \\
\overline{0,6} \\
0,1\end{array}$ \\
\hline $\begin{array}{ccccccc}\text { Asturias } & \ldots & \ldots & \ldots & \ldots & \ldots & \ldots \\
\text { Canarias } & \ldots & \ldots & \ldots & \ldots & \ldots & \ldots \\
\text { Castilla y } & \text { León } & \ldots & \ldots & \ldots & \ldots\end{array}$ & $\begin{array}{l}532,0 \\
356.9 \\
516,6\end{array}$ & $\begin{array}{l}467,1 \\
311,1 \\
460.1\end{array}$ & $\frac{40,5}{-}$ & $\frac{210,8}{-}$ & $\begin{array}{l}215,8 \\
311,1 \\
335,3\end{array}$ & $\overline{\overline{124,8}}$ & $\begin{array}{l}64,9 \\
45,8 \\
56,5\end{array}$ & $\overline{-}$ & $\overline{\overline{0,2}}$ & $\begin{array}{l}60,9 \\
32,0 \\
46,3\end{array}$ & $\begin{array}{l}0,5 \\
7,9 \\
7,6\end{array}$ & $\begin{array}{l}1,1 \\
5,9 \\
1,4\end{array}$ & $\frac{2,4}{1,0}$ \\
\hline $\begin{array}{ccccccc}\text { Galicia } & \ldots & \ldots & \ldots & \ldots & \ldots & \ldots \\
\text { Murcia } & \ldots & \ldots & \ldots & \ldots & \ldots & \ldots \\
\text { Andalucia } & \ldots & \ldots & \ldots & \ldots & \ldots \\
\text { Castilla-La } & & \text { Mancha } & \ldots & \ldots \\
\text { Extremadura } & \ldots & \ldots & \ldots & \ldots\end{array}$ & $\begin{array}{l}336,1 \\
448,8 \\
502,1 \\
372,9 \\
434,3\end{array}$ & $\begin{array}{l}291,2 \\
370,9 \\
429,8 \\
331,5 \\
393,7\end{array}$ & 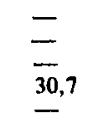 & $\begin{array}{r}73,1 \\
279,0 \\
222,2 \\
- \\
-\end{array}$ & $\begin{array}{r}176,1 \\
91,8 \\
146,4 \\
300,8 \\
393,7\end{array}$ & $\begin{array}{l}42,0 \\
61,2 \\
=\end{array}$ & $\begin{array}{l}44,9 \\
78,0 \\
72,3 \\
41,4 \\
40,7\end{array}$ & $\begin{array}{l}\frac{5,9}{7,0} \\
\frac{-}{-}\end{array}$ & $\begin{array}{l}0,2 \\
\bar{Z} \\
-\end{array}$ & $\begin{array}{l}30,1 \\
51,8 \\
47,3 \\
37,5 \\
34,0\end{array}$ & $\begin{array}{l}4,1 \\
9,6 \\
9,3 \\
2,6 \\
5,7\end{array}$ & $\begin{array}{r}4,0 \\
15,0 \\
8,3 \\
1,3 \\
0,9\end{array}$ & $\begin{array}{l}0,6 \\
1,5 \\
0,4 \\
0,1\end{array}$ \\
\hline TOTAL $4 \ldots \ldots \ldots$ & 488,2 & 419,7 & 32,0 & 139,8 & 186,9 & 61,1 & 68,4 & 1,9 & 0,6 & 45,5 & 12,1 & 7,9 & 0,5 \\
\hline
\end{tabular}

Noras: Las tasas se han calculado a partir de la población de derecho, calculada a 1 de julio de 1984, de 32.367.636 personas (excluida Cataluña). I Ordenadas según la renta per capita en 1981. Banco de Bilbao, Renta nacional de España y su distribución provincial, 1981 (Bilbao: Banco de Bilbao, 1983), p. 76. Baleares ocupa el segundo puesto en renta per capita, pero aparece dentro del segundo grupo de Comunidades Autónomas por mayor afinidad en su grado de desarrollo sociosanitario.

2 Instituciones ajenas administradas y financiadas por el INSALUD.

3 Se carece de datos para Cataluña.

- Incluye Ceuta y Melilla. No incluye Cataluña, para la que se carece de datos.

FuENTE: Instituto Nacional de la Salud, Información económico-funcional de las instituciones sanitarias, 1984. Tomo I (Madrid: INSALUD, 1986), pp. 93, 101, 109, 117 y 125 . 


\section{MAPA 2}

Nivel de desarrollo sociosanitario de las Comunidades Autónomas, en 1981

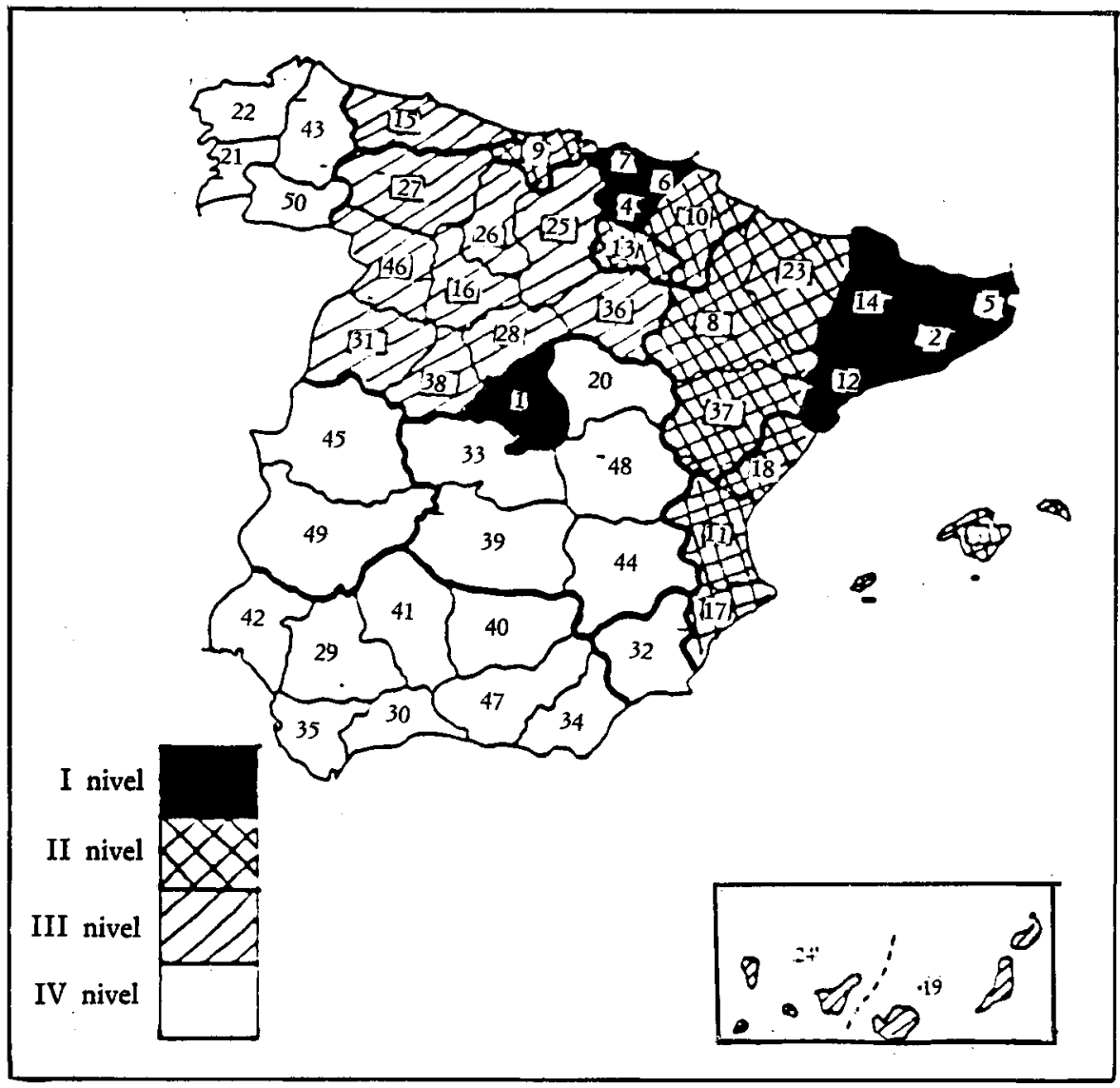

Nota: Los números indican el puesto que ocupa cada provincia según su renta per capita en orden decreciente.

Fuente: Banco de Bilbao, Renta nacional de España y su distribución provincial, 1981 (Bilbao: Banco de Bilbao, 1983), p. 103.

sonal del INSALUD), además del País Vasco y Cataluña, donde el INSALUD tiene una presencia menos importante dentro del sector sanitario y, por tanto, la dotación de personal está por debajo de la media.

El segundo grupo de Comunidades lo integran Cantabria, La Rioja, Navarra, Aragón, la Comunidad Valenciana y Baleares. Son todas ellas Comu- 
nidades de renta per capita superior a la media nacional. A excepción de Navarra (con poca presencia del INSALUD, aunque sí de otros entes públicos como la Diputación Foral), el resto de las Comunidades de este grupo tienen más recursos de personal del INSALUD que la media.

El tercer grupo lo forman Asturias, Castilla y León y Canarias, todas ellas Comunidades de desarrollo medio con (relativamente) abundante personal del INSALUD. Conviene recordar que Asturias es la Comunidad con mayor presencia del INSALUD en el sector sanitario, si se mide mediante el indicador de tasa de camas. Ciertas áreas interiores de Castilla y León tienen tasas elevadas de personal del INSALUD por habitante debido a la pérdida de población.

Todo el sur peninsular y Galicia integran el cuarto grupo de regiones. Las disparidades de dotación de recursos sanitarios del INSALUD dentro de ese grupo es reducida, aunque tanto Sevilla como Murcia, Huelva y Granada tienen tasas relativamente elevadas de personal del INSALUD. En este grupo se observa una carencia relativa tanto de personal de hospital como de personal de APS, excepto en las provincias que cuentan con ciudades sanitarias.

Se observa que, en general, la variabilidad en la dotación de recursos dentro de cada uno de los cuatro grupos de Comunidades Autónomas aumenta conforme crece el nivel de desarrollo. La distribución de camas por cada 100.000 habitantes es más desigual que la distribución de personal, lo que apoya la hipótesis de que los recursos físicos parecen peor planificados que el capital humano (De Miguel, 1978, 1985; De Miguel y Guillén, 1988). Hay, por lo general, una relación positiva entre la dotación de camas y la dotación de personal. Existen, sin embargo, Comunidades bien dotadas de camas (La Rioja, Asturias, Castilla y León) que no disponen de mucho personal, con lo que sus tasas de personal por cama son reducidas.

El Gráfico 2 permite comprobar las relaciones entre camas y personal del INSALUD. Comunidades como Asturias, Castilla y León, Baleares y La Rioja tienen muchas camas (en términos relativos), pero poco personal. En cambio Madrid, Aragón y, sobre todo, Cantabria tienen muchas camas y mucho personal por cama. Navarra y el País Vasco — con una escasa presencia del INSALUD_- son, junto con Canarias, las Comunidades con menos camas y menos personal por cama. Las cinco Comunidades menos desarrolladas tienen moderadamente pocas camas, y dotadas de poco personal, a excepción del caso de Murcia. Se observa cómo las Comunidades con menos camas del INSALUD tienen también pocas camas de otra propiedad; las excepciones a esta regla son Canarias, Cataluña y Navarra. Algunas Comunidades tienen tasas altas de camas del INSALUD y de otra propiedad: La Rioja, Aragón, Castilla y León, Baleares, País Vasco. El sur de la península y Galicia (y también la Comunidad Valenciana) no disponen de tasas elevadas de camas del INSALUD ni tampoco de otras camas. Se observa que 


\section{GRAFICO 2}

Personal y camas del INSALUD, en 1984

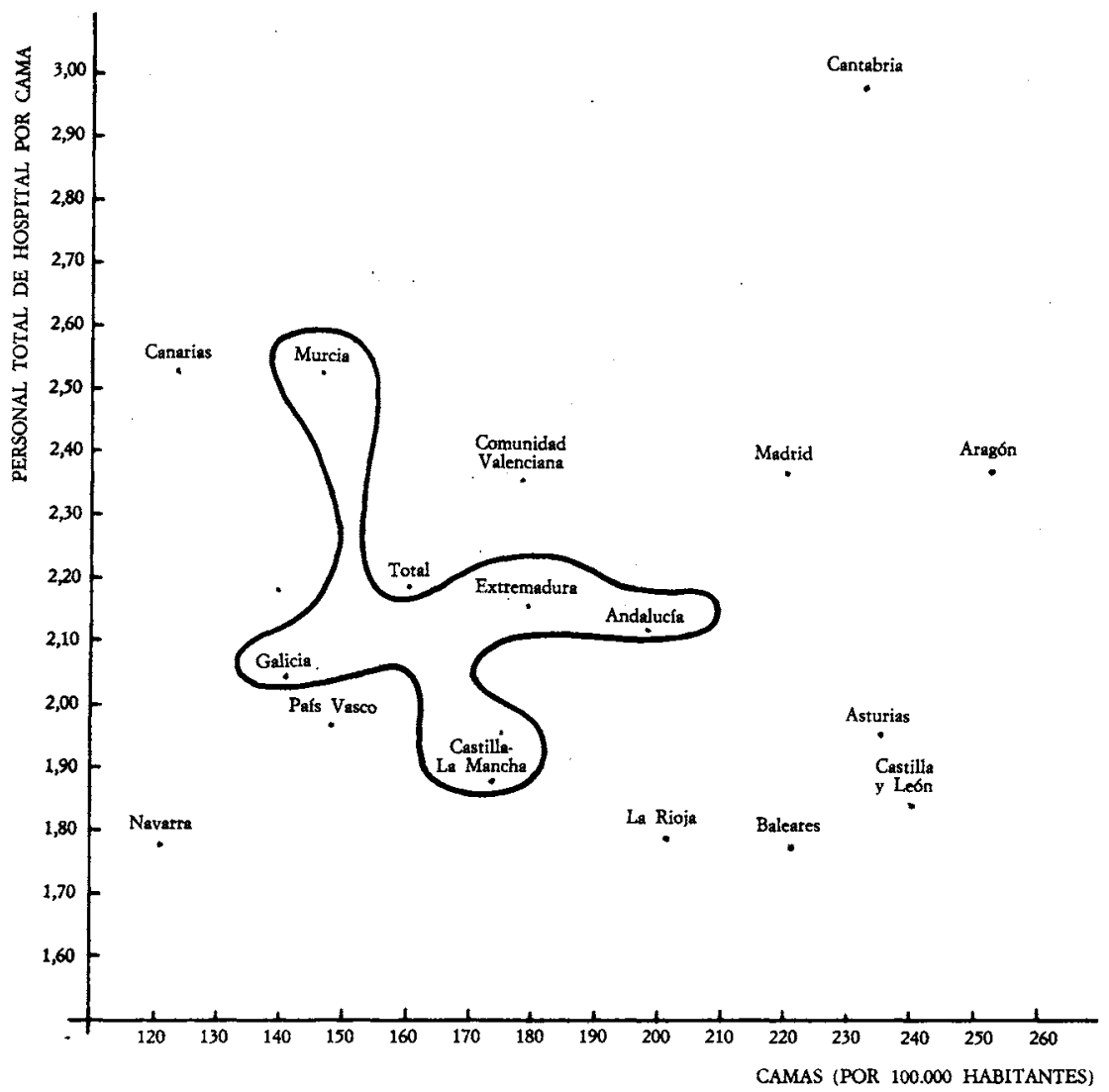

Fuente: Instituto Nacional de la Salud, Información económico-funcional de las instituciones sanitarias, 1984. Tomo II (Madrid: INSALUD, 1986), pp. 9-21 y 237-253.

la distribución de personal del INSALUD y del resto de personal se ajusta a las pautas de distribución de las camas. El mayor nivel de desarrollo está asociado positivamente con más personal hospitalario total, y también con más personal hospitalario del INSALUD. Hay, no obstante, los tres casos especiales de Navarra, Cataluña y el País Vasco, donde el sector público no es tan importante como en otras Comunidades.

El personal del INSALUD se distribuye geográficamente siguiendo las pautas de desarrollo (González, 1979), de penetración del sector público del 
INSALUD, y de la propia organización de la asistencia sanitaria (según el tipo de instituciones que predominen). Cuantas más camas hospitalarias hay y más grandes y complejos son los hospitales, más personal de enfermería y no-sanitario existe en relación al personal médico. Los procesos de especialización administrativa son también más intensos en esas zonas con ins. tituciones hospitalarias más complejas. La distribución de los recursos públicos de personal sanitario no se ajusta, pues, a la población existente en las distintas zonas, sino que presenta una situación de desigualdad.

\section{Desigualdades por Comunidades Autónomas}

Uno de los problemas del Sistema Nacional de Salud español es que el INSALUD ha distribuido históricamente sus recursos de modo heterogéneo por las Comunidades Autónomas. Las desigualdades no se producen ya sólo con respecto a la población existente, sino también respecto de las camas. Así, mientras en el conjunto del país hay dos personas trabajando en servicios hospitalarios por cada cama (1,98 en 1984), Cantabria posee 2,58, y La Rioja tan sólo 1,64. Las diferencias no parecen explicarse únicamente por el factor de desarrollo socioeconómico. Hay Comunidades que además de tener relativamente pocas camas del INSALUD tienen poco personal atendiendo esas camas: País Vasco, Navarra, Galicia y Castilla-La Mancha. Otras Comunidades tienen tasas elevadas de camas, pero dotadas de menos personal que la media (Asturias y Castilla y León).

En general, puede afirmarse que la cantidad de personal en relación al número de camas y a la población depende del tipo de instituciones sanitarias que existen en cada Comunidad. Cuanta más importancia tienen las ciudades sanitarias y los centros especiales en una Comunidad más personal existe; lo que parece demostrar la ley del imperio burocrático (Economist, 1955; Perrow, 1986; Pfeffer y Salancik, 1978). Para el total nacional, las ciudades sanitarias tienen 2,34 personas dedicadas a hospitalización por cada cama, y los centros especiales 2,16, mientras que los hospitales generales (o residencias sanitarias) tienen $1,81 \mathrm{y}$ las instituciones ajenas administradas o financiadas (IAAFI), 1,80. Esas diferencias, según el tipo de institución, se transforman luego en desigualdades más acusadas por Comunidades Autónomas a causa de la distribución espacial de los distintos tipos de instituciones: 


\begin{tabular}{|c|c|c|}
\hline Comunidad & $\begin{array}{c}\text { Tasa de personal } \\
\text { de bospitalización } \\
\text { por cama }\end{array}$ & $\begin{array}{c}\% \text { de camas } \\
\text { que pertenecen } \\
\text { a ciudades sanitarias } \\
\text { y centros especiales }\end{array}$ \\
\hline $\begin{array}{lllllllllll}\text { Madrid } & \ldots & \ldots & \ldots & \ldots & \ldots & \ldots & \ldots & \ldots & \ldots & \ldots \\
\text { País Vasco } & \ldots & \ldots & \ldots & \ldots & \ldots & \ldots & \ldots & \ldots & \ldots\end{array}$ & $\begin{array}{l}2,06 \\
1,80\end{array}$ & $\begin{array}{l}58 \\
45\end{array}$ \\
\hline 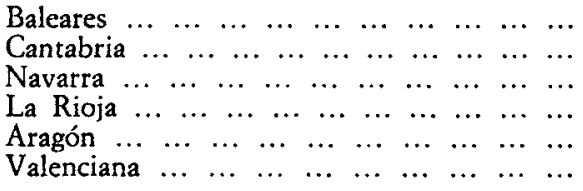 & $\begin{array}{l}2,15 \\
2,58 \\
1,70 \\
1,64 \\
2,24 \\
2,22\end{array}$ & $\begin{array}{r}0 \\
100 \\
0 \\
0 \\
40 \\
32\end{array}$ \\
\hline $\begin{array}{lllllllllll}\text { Asturias } & \ldots & \ldots & \ldots & \ldots & \ldots & \ldots & \ldots & \ldots & \ldots & \ldots \\
\text { Canarias } & \ldots & \ldots & \ldots & \ldots & \ldots & \ldots & \ldots & \ldots & \ldots & \ldots \\
\text { Castilla y } & \text { León } & \ldots & \ldots & \ldots & \ldots & \ldots & \ldots & \ldots & \ldots\end{array}$ & $\begin{array}{l}1,76 \\
2,10 \\
1,72\end{array}$ & $\begin{array}{r}58 \\
0 \\
0\end{array}$ \\
\hline $\begin{array}{lllllllllll}\text { Galicia } & \ldots & \ldots & \ldots & \ldots & \ldots & \ldots & \ldots & \ldots & \ldots & \ldots \\
\text { Murcia } & \ldots & \ldots & \ldots & \ldots & \ldots & \ldots & \ldots & \ldots & \ldots & \ldots \\
\text { Andalucía } & \ldots & \ldots & \ldots & \ldots & \ldots & \ldots & \ldots & \ldots & \ldots & \ldots \\
\text { Castilla-La } & \ddot{M}_{\text {Manch }} & \ldots & \ldots & \ldots & \ldots & \ldots & \ldots & \ldots & \ldots \\
\text { Extremadura } & \ldots & \ldots & \ldots & \ldots & \ldots & \ldots & \ldots & \ldots & \ldots \\
\end{array}$ & $\begin{array}{l}1,89 \\
2,37 \\
1,93 \\
1,74 \\
2,00\end{array}$ & $\begin{array}{r}21 \\
65 \\
46 \\
8 \\
0\end{array}$ \\
\hline $\begin{array}{lllllllllll}\text { TOTAL } & \ldots & \ldots & \ldots & \ldots & \ldots & \ldots & \ldots & \ldots & \ldots\end{array}$ & 1,98 & 35 \\
\hline
\end{tabular}

Nota: Se carece de datos para Cataluña.

La ausencia de una planificación adecuada (y de una ejecución de los planes) ha provocado que las Comunidades con instituciones con más poder de negociación o más cercanas a los centros de decisión tengan más personal por cama que el resto (Perrow, 1986; Pfeffer y Salancik, 1978). Esa pauta no es aplicable, sin embargo, a Comunidades como Asturias, Baleares y el País Vasco.

En la tabla 6 aparece la distribución del personal según el tipo de institución para el conjunto del país. Se observa que la mayor parte del personal del INSALUD trabaja en instituciones cerradas (42 personas por cada 10.000 habitantes), aunque no necesariamente en atención hospitalaria, sino también en consultas externas; existen sólo siete personas trabajando en instituciones de APS por cada 10.000 habitantes. Los centros de APS mantienen proporciones menores de personal médico que los centros hospitalarios:

\begin{tabular}{|c|c|c|}
\hline Personal & $A P S(\%)$ & Hospitales $(\%)$ \\
\hline $\begin{array}{lllllllllll}\text { Directivo } & \ldots & \ldots & \ldots & \ldots & \ldots & \ldots & \ldots & \ldots & \ldots & \ldots \\
\text { Médico } & \ldots & \ldots & \ldots & \ldots & \ldots & \ldots & \ldots & \ldots & \ldots & \ldots \\
\text { De enfermería } & \text { y } & \text { auxiliar } & \ldots & \ldots & \ldots & \ldots & \ldots \\
\text { No-sanitario } & \ldots & \ldots & \ldots & \ldots & \ldots & \ldots & \ldots & \ldots & \ldots\end{array}$ & $\begin{array}{l}0,3 \\
5 \\
54 \\
41\end{array}$ & $\begin{array}{l}0,1 \\
13^{2} \\
57 \\
31\end{array}$ \\
\hline $\begin{array}{lllllllllll}\text { Total } & \ldots & \ldots & \ldots & \ldots & \ldots & \ldots & \ldots & \ldots & \ldots\end{array}$ & 100 & 100 \\
\hline
\end{tabular}




\section{TABLA 6}

Personal del INSALUD según el tipo de institución, en 1984

(Tasas por cada 100.000 habitantes)

\begin{tabular}{|c|c|c|c|c|c|c|c|}
\hline \multirow[b]{2}{*}{ Personal } & \multirow[b]{2}{*}{ Total ${ }^{1}$} & \multirow[b]{2}{*}{$\begin{array}{l}\text { Personal } \\
\text { de APS }\end{array}$} & \multicolumn{5}{|c|}{ PERSONAL hOSPITALARIO } \\
\hline & & & Total & $\begin{array}{l}\text { Centros } \\
\text { especiales }\end{array}$ & $\begin{array}{l}\text { Ciudades } \\
\text { sanitarias }\end{array}$ & Hospitales $^{2}$ & $I A A F I^{3}$ \\
\hline $\begin{array}{llllllll}\text { Directivo } & \ldots & \ldots & \ldots & \ldots & \ldots & \ldots & \ldots \\
\text { Médico }^{4} & \ldots & \ldots & \ldots & \ldots & \ldots & \ldots & \ldots \\
\text { De enfermería }^{4} & \text { auxiliar } & \ldots & \ldots & \ldots \\
\text { No-sanitario } & \ldots & \ldots & \ldots & \ldots & \ldots & \ldots\end{array}$ & $\begin{array}{r}0,8 \\
55,6 \\
275,2 \\
156,7\end{array}$ & $\begin{array}{r}0,2 \\
3,1 \\
36,9 \\
28,2\end{array}$ & $\begin{array}{r}0,6 \\
52,5 \\
238,3 \\
128,4\end{array}$ & $\begin{array}{l}0,04 \\
3,9 \\
17,8 \\
10,3\end{array}$ & $\begin{array}{r}0,1 \\
16,1 \\
82,9 \\
40,7\end{array}$ & $\begin{array}{r}0,3 \\
22,9 \\
105,2 \\
58,5\end{array}$ & $\begin{array}{r}0,1 \\
9,6 \\
32,4 \\
10,0\end{array}$ \\
\hline 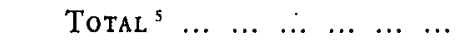 & 488,2 & 68,4 & 419,7 & 32,0 & 139,8 & 186,9 & 61,1 \\
\hline Tasa de personal por cama $\ldots \ldots$ & - & - & 1,98 & 2,16 & 2,34 & 1,81 & 1,80 \\
\hline
\end{tabular}

Notas: Las tasas se han obtenido a partir de la población de derecho, calculada a 1 de julio de 1984, de 32.367 .636 personas (excluida Cataluña).

1 Es la suma del personal hospitalario y del personal de atención primaria de salud (APS).

2 Incluye residencias sanitarias y hospitales generales.

${ }^{3}$ Instituciones ajenas administradas y financiadas por el INSALUD.

- Incluye también farmacéuticos y otros titulados superiores.

5 Incluye Ceuta y Melilla. No incluye Cataluña, para la que se carece de datos.

FUENTE: Instituto Nacional de la Salud, Información económico-funcional de las instituciones sanitarias, 1984. Tomo II (Madrid: IN. SALUD, 1986), pp. 247 y 745 . 
En principio, esas menores proporciones de personal médico parecen lógicas y van en la dirección de los criterios de la OMS (Borgoño et al., 1985; Hall y Mejía, eds., 1978; World Health Organization, 1980). Pero es sorprendente que los centros de APS tengan proporcionalmente más personal directivo y más personal no-sanitario que los hospitales, puesto que las necesidades de coordinación y control decrecen con el menor tamaño de la organización (Anderson y Warkov, 1961; Hall, 1982; Hall et al., 1967; March y Simon, 1958; Perrow, 1986; Pondy, 1969; Rushing, 1967; Terrier y Mills, 1955).

El sector del INSALUD se ha desarrollado desigualmente por las distintas Comunidades Autónomas. En algunas de ellas el sector público juega un papel complementario y minoritario respecto al sector privado hospitalario y de APS como es el caso de Navarra, País Vasco y Cataluña (De Miguel, 1978). Pero en otras Comunidades donde ya existe una dotación aceptable, como Cantabria, Aragón y Asturias, el INSALUD ha tendido a acumular más recursos, mientras en otras zonas (Galicia, Castilla-La Mancha) la dotación de personal está por debajo de la media y no existen, además, otros sectores pujantes, ya sea el privado o el de la Administración Local (Diputaciones y Cabildos). Ese desarrollo desigual se extiende también a la situación en la atención primaria y en las consultas externas de los hospitales, mostrando deficiencias en la planificación o sugiriendo la existencia de fenómenos de poder más que de búsqueda de eficiencia en la asignación de los recursos (Perrow, 1986; Pfeffer y Salancik, 1978).

\section{Atención primaria «versus» bospitales}

Los recursos presupuestarios y de personal del INSALUD se dedican primordialmente a la asistencia hospitalaria, en detrimento de la atención primaria de salud (APS); esa situación no se amolda a las recomendaciones de la Organización Mundial de la Salud (1978, 1979, 1981; Borgoño et al., 1985), ni a las declaraciones programáticas del Gobierno durante el primer cuatrienio socialista (Lluch, 1983). Varios estudios recientes han apuntado, además, la posibilidad de humanizar la asistencia y reducir los costes a través de la sustitución de recursos hospitalarios por APS (Consejo de Europa, 1980; Costas Terrones y López Casasnovas, 1985; Guillén, 1988; Lobo Aleu y Ruiz Alvarez, 1985; Puig i Junoy, 1986; Schieber, 1987). La asistencia ambulatoria del INSALUD ha dispuesto de medios humanos y de infraestructura escasos, lo que ha provocado unos tiempos medios de consulta reducidos (tres minutos por paciente), una tendencia excesiva a recetar medicamentos (provocándose un encarecimiento de la asistencia) y una tendencia a hospitalizar a pacientes que no lo requieren (Bravo et al., 1979; De Miguel, 1979; Pérez Díaz, 1982; Kelley, 1984). Esa situación acarrea dificultades 
crecientes de gestión, coordinación y control de los distintos ámbitos asistenciales del Sistema Nacional de Salud, al no cumplir la APS su función de prevención de la enfermedad, promoción de la salud y distribución de pacientes entre los distintos centros y servicios asistenciales.

El 84 por 100 del personal de asistencia sanitaria del INSALUD trabaja en centros hospitalarios, mientras el 16 por 100 restante lo hace en centros de APS. Existen, no obstante, notable diferencias por Comunidades Autónomas:

\begin{tabular}{|c|c|c|}
\hline \multirow[b]{2}{*}{ Comunidad } & \multicolumn{2}{|c|}{$\%$ DEL TOTAL DE PERSONAL } \\
\hline & En hospitales & En $A P S$ \\
\hline $\begin{array}{llllllllllll}\text { Madrid } & \ldots & \ldots & \ldots & \ldots & \ldots & \ldots & \ldots & \ldots & \ldots & \ldots & \ldots \\
\text { País Vasco } & \ldots & \ldots & \ldots & \ldots & \ldots & \ldots & \ldots & \ldots & \ldots & \ldots\end{array}$ & $\begin{array}{l}83,8 \\
80,2\end{array}$ & $\begin{array}{l}16,2 \\
19,8\end{array}$ \\
\hline 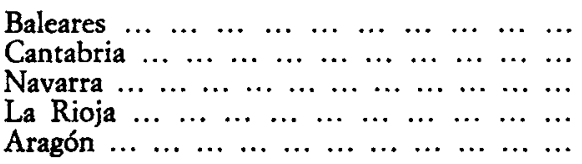 & $\begin{array}{l}86,9 \\
90,5 \\
80,3 \\
85,2 \\
88,4\end{array}$ & $\begin{array}{r}13,1 \\
9,5 \\
19,7 \\
14,8 \\
11,6\end{array}$ \\
\hline $\begin{array}{lllllllllll}\text { Valenciana } & \ldots & \ldots & \ldots & \ldots & \ldots & \ldots & \ldots & \ldots & \ldots \\
\text { Asturias } & \ldots & \ldots & \ldots & \ldots & \ldots & \ldots & \ldots & \ldots & \ldots & \ldots \\
\text { Canarias } & \ldots & \ldots & \ldots & \ldots & \ldots & \ldots & \ldots & \ldots & \ldots & \ldots \\
\text { Castilla y } & \text { León } & \ldots & \ldots & \ldots & \ldots & \ldots & \ldots & \ldots & \ldots & \ldots \\
\end{array}$ & $\begin{array}{l}86,8 \\
87,8 \\
87,2 \\
89,1\end{array}$ & $\begin{array}{l}13,2 \\
12,2 \\
12,8 \\
10,9\end{array}$ \\
\hline $\begin{array}{llllllllllll}\text { Galicia } & \ldots & \ldots & \ldots & \ldots & \ldots & \ldots & \ldots & \ldots & \ldots & \ldots & \ldots \\
\text { Murcia } & \ldots & \ldots & \ldots & \ldots & \ldots & \ldots & \ldots & \ldots & \ldots & \ldots \\
\text { Andalucía } & \ldots & \ldots & \ldots & \ldots & \ldots & \ldots & \ldots & \ldots & \ldots & \ldots \\
\text { Castilla-La } & & \text { Manch } & \ldots & \ldots & \ldots & \ldots & \ldots & \ldots & \ldots & \ldots \\
\text { Extremadura } & \ldots & \ldots & \ldots & \ldots & \ldots & \ldots & \ldots & \ldots & \ldots \\
\end{array}$ & $\begin{array}{l}86,6 \\
82,6 \\
85,6 \\
88,9 \\
90,6\end{array}$ & $\begin{array}{r}13,4 \\
17,4 \\
14,4 \\
11,1 \\
9,4\end{array}$ \\
\hline $\begin{array}{lllllllllll}\text { Total } & \ldots & \ldots & \ldots & \ldots & \ldots & \ldots & \ldots & \ldots & \ldots\end{array}$ & 83,7 & 16,3 \\
\hline
\end{tabular}

Nota: Se carece de datos para Cataluña.

Conviene tener en cuenta que no solamente realiza atención primaria el personal que trabaja en centros ambulatorios típicos, sino también el que trabaja en las consultas externas de los hospitales.

Los hospitales que mayor proporción de personal dedican a consultas externas son los centros especiales y las IAAFI ( 15 y 12 por 100 del total de personal, respectivamente). En total, un 10 por 100 del personal hospitalario del INSALUD atiende consultas externas. En algunos grandes centros hospitalarios - como en la Ciudad Sanitaria de Cruces, en Baracaldo- hasta una quinta parte del personal se ocupa de consultas externas. La variabilidad por centros y por Comunidades Autónomas es considerable: mientras en algunos hospitales apenas el 3 por 100 del personal atiende consultas externas, en otros centros la proporción sobrepasa el 15 por 100 . Una hipótesis razonable consiste en postular que esas menores proporciones de consultas 
TABLA 7

Personal de atención primaria y de consultas externas, en 1984

\begin{tabular}{|c|c|c|c|c|c|c|c|c|}
\hline \multirow[b]{2}{*}{ Comunidad Autónoma ${ }^{1}$} & \multicolumn{3}{|c|}{$\underset{Y}{\text { RESPECTO DEL TOTAL EN A.P.S. }}$} & \multicolumn{5}{|c|}{$\begin{array}{c}\% \text { DEL PERSONAL DE HOSPITALES DEDICADO } \\
\text { A CONSULTAS EXTERNAS }\end{array}$} \\
\hline & $\begin{array}{l}\text { \% personal } \\
\text { en APS } \\
\text { y consultas } \\
\text { externas }\end{array}$ & $\begin{array}{l}\text { \% personal } \\
\text { en APS }\end{array}$ & $\begin{array}{l}\% \text { personal } \\
\text { en consultas } \\
\text { externas }\end{array}$ & Total & $\begin{array}{c}\text { Centros } \\
\text { especiales }\end{array}$ & $\begin{array}{l}\text { Ciudades } \\
\text { sanitarias }\end{array}$ & $\begin{array}{l}\text { Hospitales } \\
\text { generales }\end{array}$ & $I A A F I^{2}$ \\
\hline Comunidad de Madrid $\ldots \ldots \ldots \ldots$ & 28,1 & 16,2 & 11,9 & 14,1 & 17,0 & 10,4 & 17,4 & 16,6 \\
\hline $\begin{array}{lllllllllll}\text { Cataluña } & \ldots & \ldots & \ldots & \ldots & \ldots & \ldots & \ldots & \ldots \\
\text { País Vasco } & \ldots & \ldots & \ldots & \ldots & \ldots & \ldots & \ldots & \ldots\end{array}$ & 29,0 & $\dddot{19,8}$ & $\dddot{9,2}$ & $\dddot{11,5}$ & $\ddot{-}$ & $\dddot{19,0}$ & $\dddot{3}, 9$ & $\stackrel{\cdots}{-}$ \\
\hline 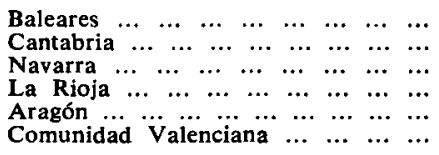 & $\begin{array}{l}16,2 \\
22,6 \\
24,5 \\
24,0 \\
17,8 \\
19,6\end{array}$ & $\begin{array}{r}13,2 \\
9,5 \\
19,7 \\
14,8 \\
11,6 \\
13,2\end{array}$ & $\begin{array}{r}3,0 \\
13,1 \\
4,8 \\
9,2 \\
6,2 \\
6,4\end{array}$ & $\begin{array}{r}3,4 \\
14,5 \\
6,0 \\
10,8 \\
7,0 \\
7,4\end{array}$ & $\begin{array}{l}\overline{14,5} \\
\overline{-} \\
=\end{array}$ & $\begin{array}{l}\bar{z} \\
\overline{5,7} \\
7,8\end{array}$ & $\begin{array}{r}3,4 \\
6,0 \\
10,8 \\
3,4 \\
7,8\end{array}$ & $\begin{array}{r}\bar{Z} \\
\overline{12,0} \\
4,4\end{array}$ \\
\hline $\begin{array}{lllllllll}\text { Asturias } & \ldots & \ldots & \ldots & \ldots & \ldots & \ldots & \ldots & \ldots \\
\text { Canarias } & \ldots & \ldots & \ldots & \ldots & \ldots & \ldots & \ldots & \ldots \\
\text { Castilla } & \text { y } & \text { León } & \ldots & \ldots & \ldots & \ldots & \ldots & \ldots\end{array}$ & $\begin{array}{l}21,6 \\
28,4 \\
19,7\end{array}$ & $\begin{array}{l}12,2 \\
12,8 \\
10,9\end{array}$ & $\begin{array}{r}9,4 \\
15,6 \\
8,8\end{array}$ & $\begin{array}{r}10,7 \\
17,9 \\
9,9\end{array}$ & $\stackrel{5,4}{-}$ & $\frac{14,3}{-}$ & $\begin{array}{r}8,1 \\
17,9 \\
8,0\end{array}$ & $\overline{15,1}$ \\
\hline $\begin{array}{llllllllll}\text { Galicia } & \ldots & \ldots & \ldots & \ldots & \ldots & \ldots & \ldots & \ldots & \ldots \\
\text { Murcia } & \ldots & \ldots & \ldots & \ldots & \ldots & \ldots & \ldots & \ldots & \ldots \\
\text { Andalucía } & \ldots & \ldots & \ldots & \ldots & \ldots & \ldots & \ldots & \ldots \\
\text { Castilla-La } & \text { Mancha } & \ldots & \ldots & \ldots & \ldots & \ldots \\
\text { Extremadura } & \ldots & \ldots & \ldots & \ldots & \ldots & \ldots & \ldots\end{array}$ & $\begin{array}{l}20,6 \\
23,5 \\
22,7 \\
20,0 \\
17,6\end{array}$ & $\begin{array}{r}13,4 \\
17,4 \\
14,4 \\
11,1 \\
9,4\end{array}$ & $\begin{array}{l}7,2 \\
6,1 \\
8,3 \\
8,9 \\
8,2\end{array}$ & $\begin{array}{r}8,4 \\
7,4 \\
9,7 \\
10,0 \\
9,0\end{array}$ & $\begin{array}{l}\bar{Z} \\
\overline{0}, 0\end{array}$ & $\begin{array}{r}16,5 \\
6,8 \\
11,4 \\
=\end{array}$ & $\begin{array}{r}5,0 \\
9,3 \\
7,0 \\
11,0 \\
9,0\end{array}$ & $\frac{8,2}{9,8}$ \\
\hline 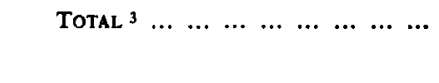 & $\begin{array}{c}22,9 \\
(158.017)\end{array}$ & $\begin{array}{c}14,0 \\
(158.017)\end{array}$ & $\begin{array}{c}8,9 \\
(158.017)\end{array}$ & $\begin{array}{c}10,3 \\
(135.849)\end{array}$ & $\begin{array}{c}14,8 \\
(10.365)\end{array}$ & $\begin{array}{c}10,9 \\
(45.245)\end{array}$ & $\begin{array}{c}8,4 \\
(60.488)\end{array}$ & $\begin{array}{c}12,5 \\
(19.751)\end{array}$ \\
\hline
\end{tabular}

Notas: Ordenadas según la renta per capita en 1981. Banco de Bilbao, Renta nacional de España y su distribución provincial, 1981 (Bilbao: Banco de Bilbao, 1983), p. 76. Baleares ocupa el segundo puesto en renta per capita, pero aparece dentro del segundo grupo de Comunidades Autónomas por mayor afinidad en su grado de desarrollo sociosanitario.

2 Instituciones ajenas administradas y financiadas por el INSALUD.

3 Incluye Ceuta y Melilla. No incluye Cataluña, para la que se carece de datos.

FUENTE: Instituto Nacional de la Salud, Información económico-funcional de las instituciones sanitarias, 1984. Tomo I (Madrid: INSALUD, 1986), pp. 109-127. 
externas en algunas Comunidades se compensan con cantidades mayores de personal empleado en centros de APS, y viceversa. Sin embargo, la tabla 7 muestra lo erróneo de esa suposición. Si se suman los porcentajes que representan el personal de APS y el personal de consultas externas respecto del total de personal se observa que existen diferencias amplias según las Comunidades Autónomas. El País Vasco — donde el INSALUD cuenta con pocos recursos propios- es la Comunidad con más personal dedicado a APS y a consultas externas (29 por 100), mientras que Baleares (16 por 100) o Aragón y Extremadura (18 por 100) son las que menos personal tienen en términos proporcionales. En el conjunto español el INSALUD asigna un 9 por 100 de su personal a consultas externas y un 14 por 100 a centros de APS, lo que suma un total de un 23 por 100. En 1984, pues, casi una cuarta parte del personal del INSALUD realiza atención primaria de salud, es decir, de carácter ambulatorio, y es la que se ocupa de la atención básica de los usuarios (Bravo et al., 1979).

Existe, sin embargo, una variabilidad considerable sin que exista una explicación aparente (las relaciones aparecen en el Grafico 3). La distribución del personal entre centros hospitalarios y centros de APS parece aleatoria, sin seguir pautas claras; salvo que un mayor nivel de desarrollo puede suponer una tasa mayor de personal de APS. Navarra (con menos personal) y Andalucía y Murcia (con más) son excepciones a esa regla. La situación es todavía más confusa si se tiene en cuenta la distribución según el personal de consultas externas y de APS (en porcentajes del total). Si la planificación hubiera sido eficaz, las Comunidades hubieran tendido a situarse sobre una recta de equilibrio entre ambos tipos de personal ( $A P S$ y consultas externas), siendo el extremo superior Cantabria (con muchas consultas externas, pero poca APS), y el inferior Navarra. Nuevamente se observa que la distribución del grupo de Comunidades Autónomas menos desarrolladas es el que mejor se adapta al modelo de equilibrio entre los dos tipos de personal.

Resulta llamativo comprobar que en dos regiones con una alta proporción de población en núcleos dispersos como Asturias y Galicia el personal de consultas externas (concentrado en grandes centros hospitalarios), sea más importante (relativamente) que el personal de APS. Un análisis similar sería aplicable a Cantabria y, sobre todo, a Canarias, donde no existen hospitales del INSALUD en todas las islas y, sin embargo, la proporción de consultas externas es relativamente elevada.

El INSALUD - que sólo posee una cuarta parte de las camas del paísdispone del 64 por 100 del personal del sector sanitario (unas 230.000 personas en 1984; ó 595 personas por cada 100.000 habitantes). Las camas del INSALUD son, junto con las del MEC, las mejor dotadas de personal. Tres cuartas partes de ese personal realiza funciones sanitarias, casi una cuarta parte es personal no-sanitario, y un 5 por 100 es personal de dirección y administración. El INSALUD ha creado, pues, un núcleo importante de 


\section{GRAFICO 3}

Personal de atención primaria de salud y consultas externas, en 1984

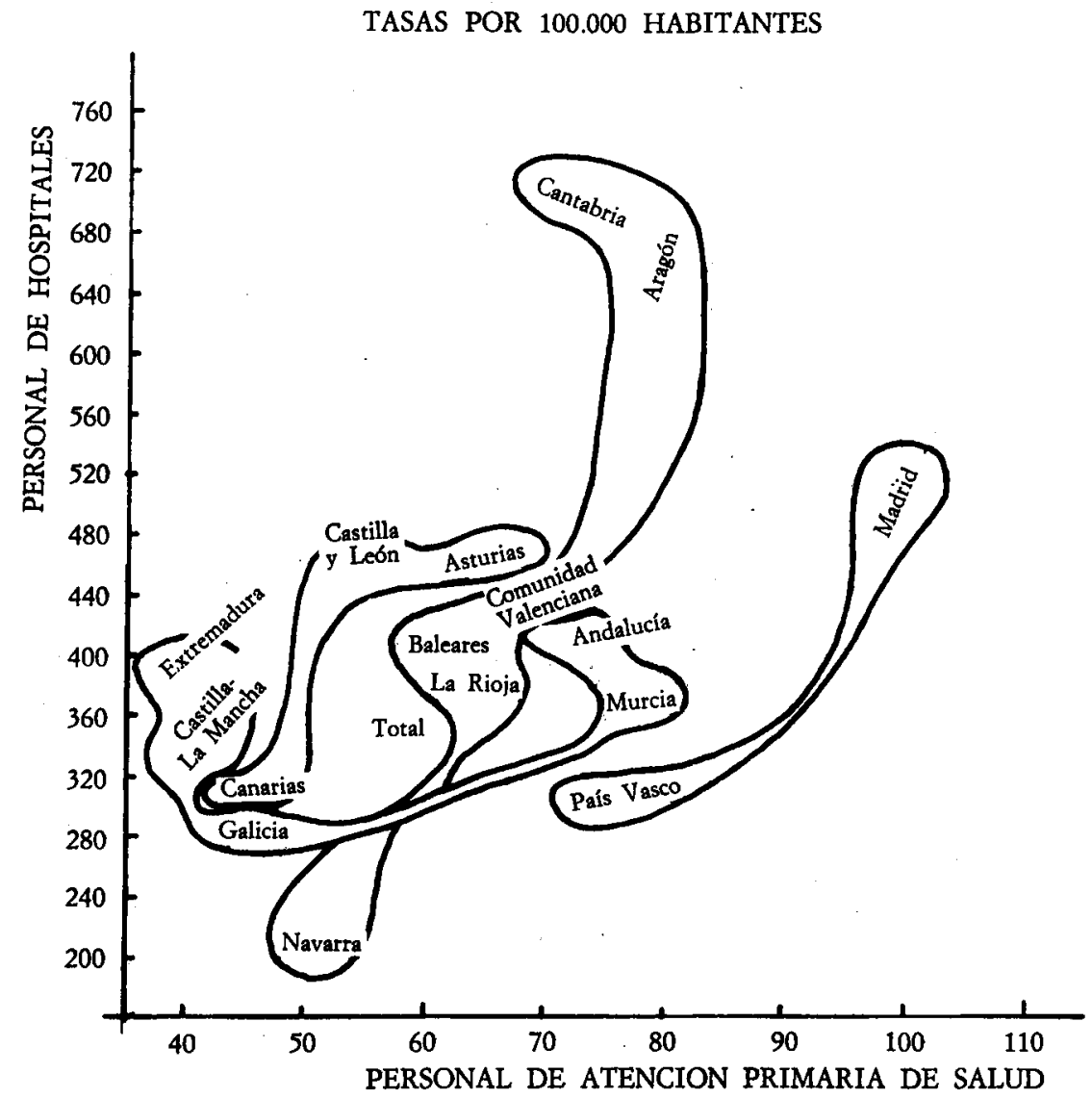

personal médico asalariado (60.000 personas) que trabajan en su mayoría ( 86 por 100 ) en organizaciones complejas. Cabe preguntarse si el INSALUD ha provocado una proletarización de la profesión médica o si, por el contrario, el poder médico se ha afianzado en el seno de las organizaciones hospitalarias (Freidson, 1986). En los hospitales del INSALUD existen más enfermeras que auxiliares de enfermería (al igual que en el MEC), lo que no ocurre en el resto del sector hospitalario. La especialización técnica de ese tipo de personal es más elevada. Se observa un proceso de especialización administrativa dentro del personal no-sanitario.

Los recursos de capital humano del INSALUD se distribuyen de modo heterogéneo por las provincias y Comunidades Autónomas, como resultado 


\section{GRAFICO 3 (Continuación)}

Personal de atención primaria de salud y consultas externas, en 1984

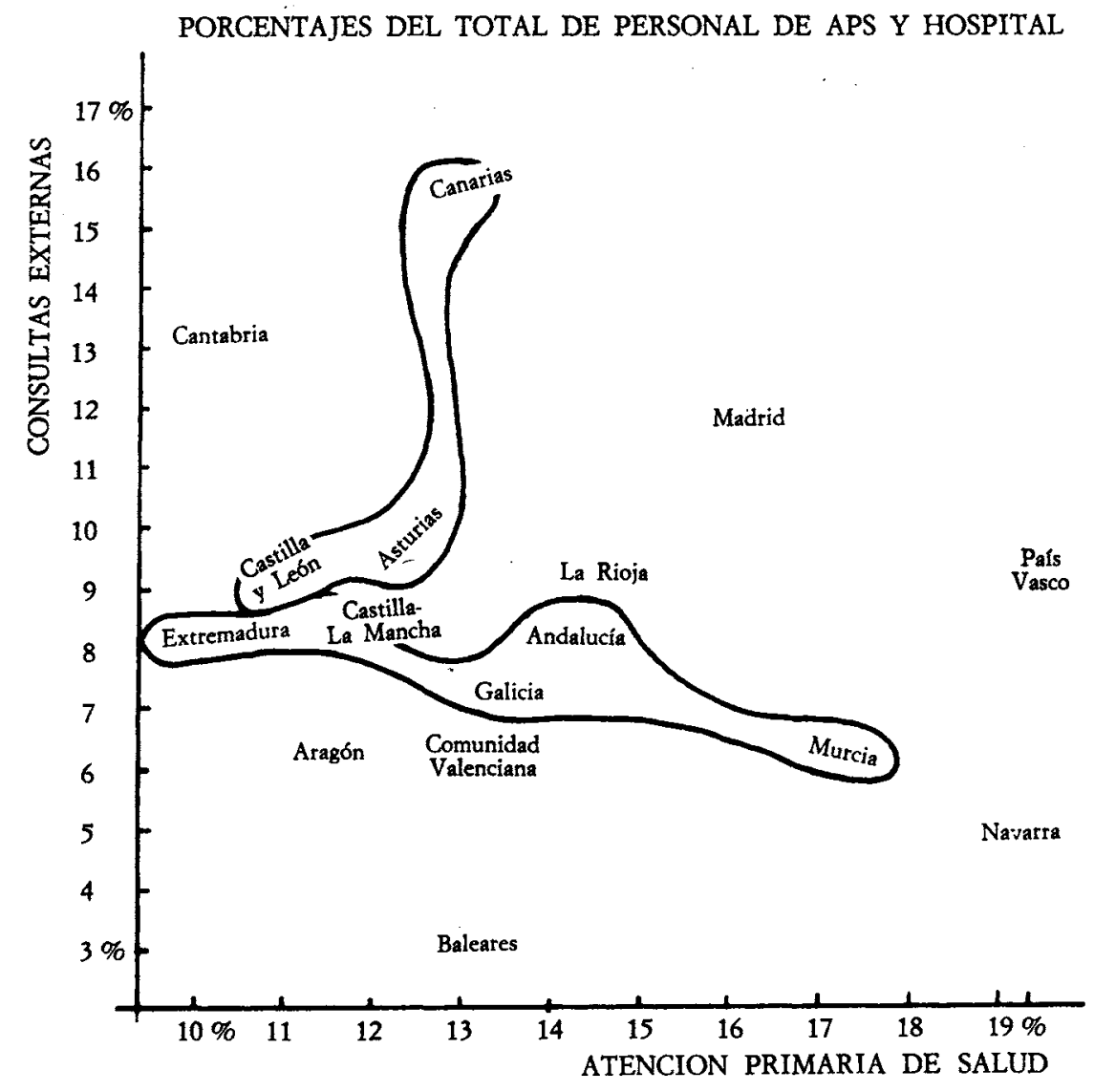

FuENTE: Instituto Nacional de la Salud, Información económico-funcional de las instituciones sanitarias, 1984. Tomo I (Madrid: INSALUD, 1986), pp. 93, 101 y 109-127.

de la acción de diversas organizaciones complejas (servicios centrales del INSALUD, ciudades sanitarias, residencias, hospitales) que no persiguen necesariamente prestar un mejor servicio a la población. La importancia del sector público del INSALUD en relación al sector privado y al de Administración Local, el grado de desarrollo socioeconómico y la dinámica poblacional inciden en la abundancia relativa de personal. Además, la propia organízación de 
la asistencia hospitalaria en ciudades sanitarias o centros especiales atrae a más personal en relación al número de habitantes, creando desequilibrios con las provincias colindantes. Se observa que hay más personal del INSALUD en las regiones más ricas y, sobre todo, menos en las más pobres.

El personal de enfermería y el personal no-sanitario abunda más que el personal médico cuanto mayor y más complejo es el sector público en una Comunidad Autónoma. La distribución de camas — que es incluso más heterogénea- no coincide con la de personal, lo que provoca que algunas Comunidades tengan muchas camas del INSALUD, pero poco personal en relación a ese número de camas. Esas situaciones de desigualdad son importantes en los años ochenta, cuando se proyectan realizar las transferencias de competencias en materia de sanidad del Estado a las Comunidades Autónomas.

El INSALUD no ha conseguido llevar a la práctica una política aceptable de APS. Tan sólo un 14 por 100 de su personal trabaja en centros de atención primaria, aunque un 9 por 100 adicional realiza consultas externas en hospitales. Ese sistema dual crea una concentración de personal de APS en núcleos concretos, y está desequilibrado, pues algunas Comunidades poseen hasta el doble de personal dedicado a atención ambulatoria que otras.

El Sistema Nacional de Salud se enfrenta, pues, a finales de la década de los ochenta con un proceso de descentralización de competencias en el que influye poderosamente una situación de partida heterogénea según las Comunidades Autónomas, y con una manifiesta insuficiencia de recursos destinados a atención primaria. La hipótesis más importante es que en los últimos años (y décadas) la dinámica del sector público y de las profesiones sanitarias ha contribuido a que las desigualdades sanitarias, según las Comunidades Autónomas, sean cada vez mayores, lo que supone una contradicción con las tendencias isomórficas apuntadas por DiMaggio y Powell (1983). Los Servicios de Salud autonómicos pueden resultar, pues, en un modelo desigual, difícil de coordinar y controlar.

\section{BIBLIOGRAFIA}

ANDERSON, T., y WARKov; S. (1961): «Organizational size and functional complexity», Administrative Science Quarterly, 26 (1): 23-28.

Bravo, Federico; De Miguel, Jesús M.; Polo, Roberto; Reventós, Jacint, y Rodríguez, Josep A. (1979): Sociología de los ambulatorios: Análisis de la asistencia sanitaria en la Seguridad Social, Barcelona: Ariel.

Borgoño, J. M., et al. (1985): Necesidades de personal sanitario para alcanzar la salud para todos en el año 2000 mediante la atención primaria, Ginebra: Organización Mundial de la Salud.

Consejo DE EuRopa (1980): The Cost of Health Care in Member States of the Council of Europe and in Finland, Estrasburgo: Council of Europe.

Costas Terrones, J. C., y López Casasnovas, G. (1985): «La expansión del gasto sanitario y la Ley General de Sanidad», Papeles de Economia Española, 23: 194-202. 
De Mrguel, Jesús M. (1976): La reforma sanitaria en España: El capital bumano en el sector sanitario, Madrid: Cambio 16.

- (1978): «El teorema de Watson del sector sanitario: Hacia una teoría sociológica de la Seguridad Social española», Papers: Revista de Sociología, 10: 115-145.

- (1979): La sociedad enferma: Las bases sociales de la politica sanitaria española, Madrid: Akal.

- (1985): La salud pública del futuro, Barcelona: Ariel.

- (1986): «El sector público sanitario», Sistema, 75: $29-61$.

- y GuilléN, Mauro F. (1988): «The health system in Spain», en Mark G. Field (ed.), Success and Crisis in Healtb Care Systems: A Cross-National Approacb, Londres: Tavistock (en prensa).

DiMaggio, Paul J., y Powell, Walter W. (1983): «The Iron Cage revisited: Institutional isomorphism and collective rationality in organizational fields», American Sociological Review, 48: 147-160.

Economist, The (1955): «Parkinson's Law», The Economist (19 noviembre): 635-637.

FreIdson, Eliot (1986): Professional Powers: A Study of the Institutionalization of Formal Knowledge, Chicago y Londres: The University of Chicago Press.

González, Benjamín (1979): El capital bumano en el sector sanitario: La distribución de médicos en España, Madrid: Centro de Investigaciones Sociológicas.

GuilléN, M. F. (1988): «Crisis y capital humano sanitario: Análisis comparativo de la eficiencia de los sistemas de salud», Información Comercial Española. Revista de Economia, 660-661 (agosto-septiembre): 221-243.

Hall, Richard H. (1982): Organizaciones: Estructura y proceso, Madrid: Prentice-Hall Internacional.

- et al. (1967): «Organizational size, complexity, and formalization», American Sociological Review, 32 (6): 903-912.

Hall, T. L., y Mejía, A. (eds.) (1978): Health Manpower Planning: Principles, Methods, Issues, Ginebra: WHO.

Instituto Nacional de la Salud (1986): Memoria 1984, Madrid: Instituto Nacional de la Salud.

- (1985): «El personal sanitario de los hospitales del INSALUD: Situación actual», Bole tín de Indicadores Sanitarios, 3 (3): 16-22.

- (1986): Información económico-funcional de las instituciones sanitarias, 1984, Madrid: Instituto Nacional de la Salud, 2 vols.

KeLleY, J. B. (1984): «Health care in the Spanish social security system: Public-private relationships», International Journal of Healtb Services, 14 (2): $309-320$.

Lluch, Ernest (1983): Politica general del Ministerio de Sanidad y Consumo, Madrid: Ministerio de Sanidad y Consumo.

Lobo Aleu, F., y Ruiz Alvarez, J. L. (1985): «La crisis y el ajuste estructural en el sector de la salud", Papeles de Economia Española, 23: 179-193.

March, James G., y Simon, Herbert A. (1958): Organizations, Nueva York: John Wiley. Existe traducción al castellano en Barcelona: Ariel, 1986.

Nadal, Julio (ed.) (1984): Oferta y demanda de médicos en España: Una primera aproximación, Madrid: Ministerio de Sanidad y Consumo.

OECD (1985): Measuring Health Care 1960-1983: Expenditure, Costs, and Performance, París: OECD.

Organización Mundial de la Salud (1978): Alma-Ata 1978: Atención Primaria de Salud, Ginebra: Organización Mundial de la Salud.

- (1979): Formulación de estrategias con el fin de alcanzar la salud para todos en el año 2000: Principios básicos y cuestiones esenciales, Ginebra: Organización Mundial de la Salud.

- (1981): Estrategia mundial de salud para todos en el año 2000, Ginebra: Organización Mundial de la Salud.

Pérez Díaz, V. (1982): «Médicos, administradores y enfermos: La calidad de la asistencia sanitaria», Papeles de Economía Española, 12-13: 231-251.

Perrow, Charles (1986): Complex Organizations: A Critical Essay, 3. ed., Nueva York: Random House.

PfefFER, Jeffrey, y SAlancik, Gerald R. (1978): The External Control of Organizations: $A$ Resource Dependence Perspective, Nueva York: Harper and Row. 
Pondy, L. R. (1969): «Effects of size, complexity and ownership on administrative intensity», Administrative Science Quarterly, 14 (1): 47-60.

PUig I JUNoY, J. (1986): "Gasto hospitalario y políticas de contención de costes", Cua. dernos de Economia, 14: 273-300.

Rushing, W. A. (1967): "The effects of industry size and division of labor on administration", Administrative Science Quarterly, 12 (2): 273-295.

SCHIEBER, George J. (1987): Financing and Delivering Health Care: A Comparative Analysis of OECD countries, París: OECD.

Terrier, F. C., y MiLls, D. C. (1955): "The effect of changing size upon the internal structure of an organization», American Sociological Review, 20 (1): 11-14.

World Health Organization (1980): Sixth Report on the World Health Situation. 2 vols., Ginebra: WHO. 
NOTAS DE INVESTIGACION 